\title{
Nonlocality free wirings and the distinguishability between Bell boxes
}

\author{
Rodrigo Gallego \\ Dahlem Center for Complex Quantum Systems, Freie Universität Berlin, 14195 Berlin, Germany
}

\author{
Leandro Aolita \\ Instituto de Física, Universidade Federal do Rio de Janeiro, P. O. Box 68528, Rio de Janeiro, RJ 21941-972, Brazil \\ and International Institute of Physics, Federal University of Rio Grande do Norte, 59070-405 Natal, Brazil
}

(Received 6 January 2017; published 17 March 2017)

\begin{abstract}
Bell nonlocality can be formulated in terms of a resource theory with local-hidden variable models as resourceless objects. Two such theories are known, one built upon local operations assisted by shared randomness (LOSRs) and the other one allowing, in addition, for prior-to-input classical communication. We show that prior communication, although unable to create nonlocality, leads to wirings not only beyond LOSRs but also not contained in a much broader class of (nonlocality-generating) global wirings. Technically, this is shown by proving that it can improve the statistical distinguishability between Bell correlations optimized over all fixed measurement choices. This has implications in nonlocality quantification, and leads us to a natural universal definition of Bell nonlocality measures. To end up with, we also consider the statistical strength of nonlocality proofs. We point out some issues of its standard definition in the resource-theoretic operational framework, and suggest simple fixes for them. Our findings reveal nontrivial features of the geometry of the set of wirings and may have implications in the operational distinguishability of nonlocal behaviors.
\end{abstract}

DOI: 10.1103/PhysRevA.95.032118

\section{INTRODUCTION}

Bell nonlocality is an exotic quantum phenomenon by which correlations between the outcomes of spacelike separated measurements cannot be explained by local hiddenvariable theories, i.e., by any classical model relaying exclusively on past common causes [1,2]. Apart from their fundamental implications, such nonlocal correlations have been identified as a valuable resource for practical informationtheoretic tasks, such as quantum key distribution [3-5], perfect-randomness expansion [6,7] and [8,9] amplification, or distributed computations [10], for example. These protocols exploit the nonlocal correlations shared between distant users as physical resources, and process their generated classical information locally, with the aid of a priori communicated bits or shared randomness. In these scenarios, Bell nonlocality plays the role of an operational resource for classicalinformation processing, i.e., one that can be composed, acted on, and transformed between its different forms, depending on one's needs [11-14]. This is formalized by so-called resource theories of Bell nonlocality [15-18].

Resource theories constitute powerful formalisms in quantum information for the abstract treatment of a physical property as an operational resource. They have been built also for other types of quantum nonlocality, such as entanglement [19] and Einstein-Podolski-Rosen steering [20], as well as athermal states [21], quantum coherence [22], and several other notorious properties of quantum systems (see, e.g., Ref. [23] and references therein). A resource theory identifies a set of mathematical objects-describing states of the physical system under scrutiny, a subset thereof of uninteresting objects-composed of the states without the property considered the resource in question, and a class of free operations - consisting of physical transformations under which the resourceless subset is closed. For Bell nonlocality, two classes of free operations are well studied: one restricted to local operations assisted by shared randomness (LOSR) $[17,18]$ and the other allowing also for communication that contains no information about the measurement settings of the transformed object, called wirings and prior-to-input classical communication (WPICCs) [15]. Until this work, it was not clear whether these two classes displayed any difference. In fact, they have sometimes been referred to as the same single class $[17,18]$. Here, we clarify this question showing that the classes are actually inequivalent.

On the other hand, central to any resource theory is the distinguishability between the objects considered, and, in particular, the distinguishability between resourceful and resourceless ones. The distinguishability between Bell correlations was studied in Ref. [24]. There, a measure of statistical distinguishability between the measurement outcomes of a given nonlocal device and any local one, called the statistical strength of nonlocality proofs, was put forward. However, such measure was derived from a game-theoretic perspective and consistency with resource-theoretic operational approaches to nonlocality was not checked for.

Here, we study the interconnection between the two main resource theories of Bell nonlocality, based on the paradigms of LOSRs and WPICCs, in view of the operational task of distinguishing Bell boxes. To begin with, we derive an explicit parametrization for a generic WPICC map. Then, we show that WPICCs can be used to increase the statistical distinguishability between two Bell boxes. This is, in contrast, impossible not only with LOSRs, but also with the more general global wirings (GWs), defined analogous to LOSRs but with arbitrary nonlocal correlations playing the role of shared randomness. Technically, this is proven by showing that the relative entropy between Bell-box behaviors increases under WPICCs, while it can only decrease under all GWs.

We then move on to study the quantification of Bell nonlocality with respect to the two different resource theories. In particular, we show that every nonlocality measure with 
respect to LOSRs is also a valid measure with respect to WPICCs, and that the converse holds if the quantifier satisfies the natural requirement of convexity. This leads us to a universal definition of Bell nonlocality monotones, consistent with both resource theories. As an example of such monotone, we provide a definition of the relative entropy of nonlocality in terms of the relative entropy between behaviors. Finally, we probe the three variants, introduced in Ref. [24], of the statistical strength of nonlocality proofs as Bell nonlocality monotones. One of them coincides with the relative entropy of nonlocality and is, therefore, automatically a nonlocality monotone. We prove that, from the other two, one is a Bell nonlocality monotone, whereas the other one is not even monotonous under LOSRs. We end up by providing physical arguments by which, from the two variants defining satisfactory nonlocality measures, we find one better than the other as quantifier of the statistical strength of nonlocality proofs, even from a game-theoretic perspective.

The paper is structured as follows. In Sec. II we introduce the basic notions and notation. In Sec. III we discuss the different classes of transformations between Bell boxes. An explicit analytic expression for generic WPICCs is provided there too. In Sec. IV we prove that the relative entropy between behaviors can increase under WPICCs, while it can only decrease under GWs. In Sec. V we study generic Bell nonlocality monotones and, in particular, the relative entropy of nonlocality. In Sec. VI we revisit statistical strength of nonlocality proofs from a resource-theoretic perspective. We finish the paper in Sec. VII with a few relevant final remarks.

\section{PRELIMINARIES}

In this section we introduce the basics of Bell nonlocality [2]. We consider two spacelike separated experimenters, Alice and Bob, who make local measurements on a bipartite system composed of two black-box devices. Alice's box has $x \in\left[s_{A}\right]$ measurement settings (inputs) and $a \in\left[r_{A}\right]$ measurement results (outputs) and, similarly, Bob's box admits $y \in\left[s_{\mathrm{b}}\right]$ inputs and returns $b \in\left[r_{B}\right]$ outputs, where $s_{A}, r_{A}, s_{\mathrm{b}}, r_{B} \in \mathbb{N}$, and the notation $[n]:=\{0, \ldots, n-1\}$, for any $n \in \mathbb{N}$, has been introduced. For notational simplicity, but without loss of generality, we take $s:=s_{A}=s_{B}$ and $r:=r_{A}=r_{B}$. The experiment is described by a normalized bipartite conditional probability distribution

$$
\boldsymbol{P}:=\{P(a, b \mid x, y)\}_{a, b \in[r], x, y \in[s]},
$$

where $P(a, b \mid x, y)$ is the conditional probability of obtaining the output values $a$ and $b$ given that the input values $x$ and $y$. We refer to any normalized bipartite conditional probability distribution as a black-box behavior or, simply, behavior, for short.

Since the measurements constitute spacelike separated events, the statistics must fulfill the no-signaling principle, given by the well-known conditions

$$
\begin{aligned}
& P(b \mid y)=\sum_{a^{\prime}} P\left(a^{\prime}, b \mid x^{\prime}, y\right) \quad \forall x^{\prime} \in[s], \\
& P(a \mid x)=\sum_{b^{\prime}} P\left(a, b^{\prime} \mid x, y^{\prime}\right) \quad \forall y^{\prime} \in[s],
\end{aligned}
$$

for all $a, b \in[r]$ and $x, y \in[s]$. That is, the marginal conditional distribution $\{P(a \mid x, y)\}_{a \in[r], x, y \in[s]}$ $\left[\{P(a \mid x, y)\}_{b \in[r], y \in[s]}\right]$ for Alice (Bob) should not depend on Bob's (Alice's) measurement choice. We refer to the set of all behaviors that fulfill the linear constraints (2) as NS. In addition, we denote by $Q$ the set of all quantum behaviors, i.e., all those that can be expressed as

$$
P(a, b \mid x, y)=\operatorname{Tr}\left[\varrho_{A B} M_{x}^{a} \otimes M_{y}^{b}\right]
$$

where $\varrho_{A B}$ is a bipartite quantum state and $M_{x}^{a}$ and $M_{y}^{b}$ are local measurement operators corresponding to Alice and Bob, respectively. In turn, we call $L$ the set of all local behaviors, i.e., all those for which there exists a normalized probability distribution $\boldsymbol{P}_{\Lambda}$ and two normalized conditional probability distributions $\boldsymbol{P}_{A \mid X, \Lambda}$ and $\boldsymbol{P}_{B \mid Y, \Lambda}$ such that

$$
P(a, b \mid x, y)=\sum_{\lambda} P_{\Lambda}(\lambda) P_{A \mid X, \Lambda}(a \mid x, \lambda) P_{B \mid Y, \Lambda}(b \mid y, \lambda),
$$

for all $a, b \in[r]$ and $x, y \in[s]$. The variable $\lambda$ is called a local-hidden variable and the decomposition (4) is accordingly referred to as a local-hidden variable (LHS) model for the behavior. It is a well-known fact that

$$
\mathrm{L} \subset \mathrm{Q} \subset \mathrm{NS} \text {. }
$$

Finally, we say that any $\boldsymbol{P} \notin \mathrm{L}$ is a nonlocal behavior, and refer to this fact as nonlocality.

\section{NONLOCALITY AS AN OPERATIONAL RESOURCE}

Here, we focus on nonlocality as an operational resource for information-theoretic tasks. This is formalized by socalled resource theories $[15,17,18,20]$. Resource theories are composed of three main elements: (i) mathematical objects describing the system under scrutiny, (ii) a particular property of such objects considered the valuable resource, and (iii) a class of free operations for the resource, i.e., physical transformations fulfilling the essential requirement of mapping all free objects (i.e., those without the resource) into free objects. For Bell nonlocality, these three components are (i) behaviors $\boldsymbol{P} \in \mathrm{NS}$, (ii) nonlocality, i.e., that $\boldsymbol{P} \notin \mathrm{L}$, and (iii) physical transformations under which $L$ (the set of free objects) is closed, i.e., that do not create nonlocality.

Importantly, the requirement that the free operations leave $L$ invariant is necessary but not sufficient to specify the concrete class of free operations. Typically, this specification is made on the basis of the physical restrictions native of the scenario where nonlocality serves as a resource. Consequently, in general, there can be multiple classes of free operations, and, therefore, of resource theories, for the same resource, as we discuss next.

\section{A. Two resource theories of Bell nonlocality}

We restrict throughout to the paradigm of linear maps from behaviors into behaviors. Each such transformation can be physically realized by wiring inputs and outputs of the initial black-box measurement devices with the inputs and outputs of other black boxes [25,26]. Hence, from now on, we refer to any linear black-box transformation as a wiring. 
Within a resource theory of nonlocality $[17,18]$, a wiring $\mathcal{W}$ is a free operation for nonlocality if

$$
\boldsymbol{P}_{\mathrm{f}}=\mathcal{W}\left(\boldsymbol{P}_{0}\right) \in \mathrm{L}, \forall \boldsymbol{P}_{0} \in \mathrm{L} .
$$

$\boldsymbol{P}_{\mathrm{f}}:=\left\{P_{\mathrm{f}}(\alpha, \beta \mid \chi, \psi)\right\}_{\alpha, \beta \in\left[r_{f}\right], \chi, \psi \in\left[s_{f}\right]}$ represents the final behavior after the transformation $\mathcal{W}$ on an initial behavior $\boldsymbol{P}_{0}$ of the form (4), where $P_{\mathrm{f}}(\alpha, \beta \mid \chi, \psi)$ is the conditional probability of obtaining the output values $\alpha$ and $\beta$ given that the input values are $\chi$ and $\psi$ for the final box. Note that, in full generality, we allow the cardinality of the alphabets of inputs and outputs to change (from $s$ and $r$ to $s_{f}$ and $r_{f}$, respectively). We refer to any wiring that is a free operation for nonlocality as a nonlocality-free wiring, or, for short, simply a free wiring. There are two classes of free wirings known.

\section{Local operations and shared randomness}

The first class is called local operations assisted by shared randomness (see, e.g., Ref. [17] for a review on the topic). This class, which we denote as LOSR, encodes the physical restriction that Alice and Bob can only process the classical information available locally, without any communication between them. It is composed of all wirings $\mathcal{W}_{\text {LOSR }}$ explicitly parametrized by $\boldsymbol{P}_{\mathrm{f}}=\mathcal{W}_{\text {LOSR }}\left(\boldsymbol{P}_{0}\right)$, with

$$
\begin{aligned}
P_{\mathrm{f}}(\alpha, \beta \mid \chi, \psi):= & \sum_{a, b, x, y} O^{(\mathrm{L})}(\alpha, \beta \mid a, b, x, y, \chi, \psi) \\
& \times P_{0}(a, b \mid x, y) \times I^{(\mathrm{L})}(x, y \mid \chi, \psi),
\end{aligned}
$$

where $\boldsymbol{I}^{(\mathrm{L})}$ and $\boldsymbol{O}^{(\mathrm{L})}$ are arbitrary boxes in $\mathrm{L}$. That is, the input and output dits of the initial box $\boldsymbol{P}_{0}$ are processed (wired) locally, as sketched in Fig. 1(a), with (well-normalized) input and output behaviors $\boldsymbol{I}^{(\mathrm{L})}$ and $\boldsymbol{O}^{(\mathrm{L})}$ that admit both a LHV model, to produce the input and output dits of the final box $\boldsymbol{P}_{\mathrm{f}}$. It is known that if $\boldsymbol{P}_{0} \in \mathrm{L}$ then $\mathcal{W}_{\mathrm{LOSR}}\left(\boldsymbol{P}_{0}\right) \in \mathrm{L}$, if $\boldsymbol{P}_{0} \in \mathrm{Q}$ then $\mathcal{W}_{\mathrm{LOSR}}\left(\boldsymbol{P}_{0}\right) \in \mathrm{Q}$, and if $\boldsymbol{P}_{0} \in \mathrm{NS}$ then $\mathcal{W}_{\mathrm{LOSR}}\left(\boldsymbol{P}_{0}\right) \in$ NS [13]. Examples of wirings in LOSR are local relabelings of inputs or outputs and mixing with a local behavior [17].

\section{Wirings and prior to inputs classical communication}

The second class is commonly known as wirings and prior-to-input classical communication [27]. This is the class WPICC of all wirings $\mathcal{W}_{\text {WPICC }}$ operationally defined by the following two-stage sequence [see Fig. 1(b)] [15].

(1) Preparation phase. Alice and Bob are allowed to use the initial box, i.e., to choose $x$ and/or $y$, generating $a$ and/or $b$, respectively, and to communicate $x, y, a, b$, or any other random bit of their choice, before the final inputs $\chi$ and $\psi$ are chosen.

(2) Measurement phase. Once $\chi$ and $\psi$ are chosen, Alice and Bob apply a generic LOSR wiring.

This sequence unambiguously defines the class WPICC. In Appendix A, we provide an explicit parametrization of generic wirings in the class. The analytic expression obtained is somewhat cumbersome due to the many different options that branch off during the preparation phase, but it can be written in a simplified form as

$$
\mathcal{W}_{\text {WPICC }}\left(\boldsymbol{P}_{0}\right):=p L\left(\boldsymbol{P}_{0}\right)+(1-p) \mathcal{W}_{\text {LOSR }}\left(\boldsymbol{P}_{0}\right),
$$

where $0 \leqslant p \leqslant 1, \mathcal{W}_{\text {LOSR }} \in \mathrm{LOSR}$, and $L$ is a linear map from NS to L, i.e., $L\left(\boldsymbol{P}_{0}\right) \in \mathrm{L}$ for all $\boldsymbol{P}_{0} \in \mathrm{NS}$. From Eq. (8), one immediately sees that if $\boldsymbol{P}_{0} \in \mathrm{L}$ then $\mathcal{W}_{\text {WPICC }}\left(\boldsymbol{P}_{0}\right) \in \mathrm{L}$ and if $\boldsymbol{P}_{0} \in \mathrm{NS}$ then $\mathcal{W}_{\text {WPICC }}\left(\boldsymbol{P}_{0}\right) \in \mathrm{NS}$. Besides, it clearly (a)

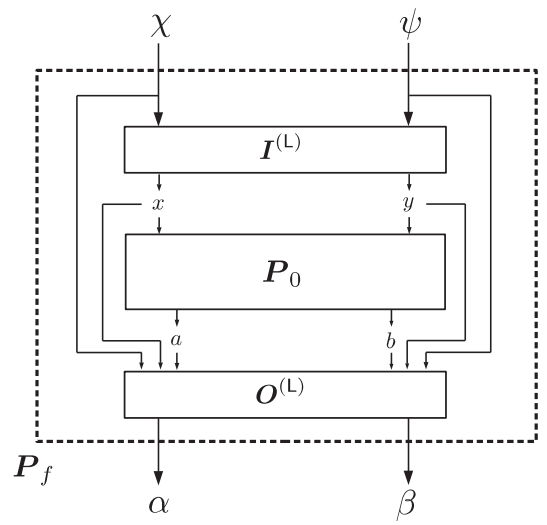

(b)

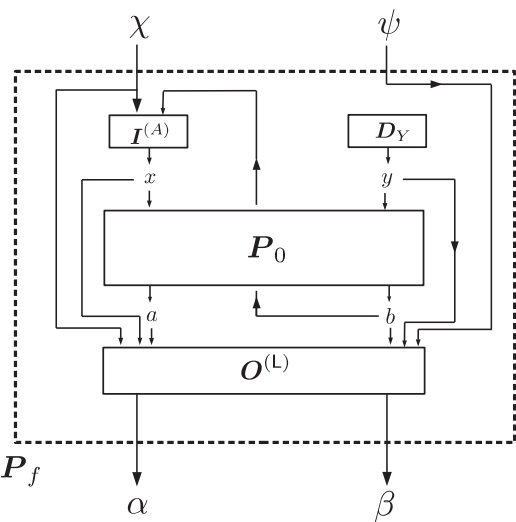

(c)

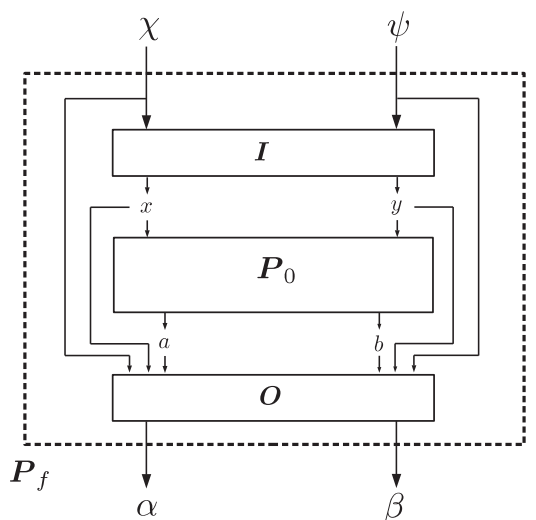

FIG. 1. Circuit representation of the three main types of Bell-box wirings. Panel (a) shows a generic LOSR wiring. There, the inputs of the final box $\boldsymbol{P}_{\text {f }}$ are processed by an input box with local behavior $\boldsymbol{I}^{(L)}$, whose outputs are input to the initial box $\boldsymbol{P}_{0}$. The outputs produced by $\boldsymbol{P}_{0}$ are, in turn, processed by an output box with local behavior $\boldsymbol{O}^{(L)}$, which has as inputs all previously generated dits, without any exchange of dits between Alice and Bob's sides. The outputs of $\boldsymbol{O}^{(L)}$ are the final outputs. Panel (b) shows an example of the class WPICC, which allows for communication between the users provided it does not carry any information about the final inputs. In the example, Bob measures his initial local box before his final input is decided. He chooses his initial input with a single-partite box $\boldsymbol{D}_{Y}$ without inputs. The output of his initial box is sent to Alice, who uses it, together with her final input, to choose her initial input according to a single-partite behavior $\boldsymbol{I}^{(A)}$. The remaining dit processing is the same as in a LOSR wiring. Finally, in panel (c), a generic GW wiring is displayed. The circuitry there is analogous to that of LOSR wirings, except that the input and output boxes are governed by generic nonlocal behaviors $\boldsymbol{I}$ and $\boldsymbol{O}$, respectively, not restricted to L. In fact, $\boldsymbol{I}$ and $\boldsymbol{O}$ need in general not even be in NS. Important subclasses of GW are the no-signaling and quantum wirings, obtained when $\boldsymbol{I}$ and $\boldsymbol{O}$ are restricted to NS and Q, respectively. While LOSR and WPICC are classes of nonlocality-free wirings, GW can create Bell nonlocality, i.e., it can map local behaviors into nonlocal ones. 
holds that

$$
\mathrm{LOSR} \subseteq \text { WPICC }
$$

However, as we show in Corollary 4 in Sec. IV B, the reciprocal turns out not to be true.

As a final but important remark, we note that, if $\boldsymbol{P}_{0} \notin$ NS, causal loops can arise due to the preparation phase. For instance, in the second panel of Fig. 1, the initial output $b$ is used to choose the initial input $x$. This can clearly introduce a causal loop if $\boldsymbol{P}_{0}$ is signaling from Alice to Bob. If, on the contrary, $\boldsymbol{P}_{0} \in \mathrm{NS}$, such problems are avoided and the preparation phase is consistent. Hence, throughout, we restrict the domain of WPICC wirings to the set NS of no-signaling behaviors. See Appendix A for more details about WPICC.

\section{B. Nonlocal wirings}

Next, we consider a third class of wirings, which we call global wirings and denote by GW. GW is not a class of free operations for Bell nonlocality, but it is relevant to the results we discuss below. The class is composed of all the wirings $\mathcal{W}_{\text {GW }}$ that act globally on the input and output dits, without any restriction of locality or even no-signaling. They process $\boldsymbol{P}_{0}$ as an effective single-partite distribution with the inputs $(x, y)$ and outputs $(a, b)$ treated as higher-dimensional single-partite inputs and outputs, respectively. They are defined as the LOSR wirings but with generic (instead of local) boxes wired to the input and output dits of the initial box, explicitly parametrized by $\boldsymbol{P}_{\mathrm{f}}=\mathcal{W}_{\mathrm{GW}}\left(\boldsymbol{P}_{0}\right)$, with

$$
\begin{aligned}
P_{\mathrm{f}}(\alpha, \beta \mid \chi, \psi):= & \sum_{a, b, x, y} O(\alpha, \beta \mid a, b, x, y, \chi, \psi) \\
& \times P_{0}(a, b \mid x, y) \times I(x, y \mid \chi, \psi),
\end{aligned}
$$

with $\boldsymbol{I}$ and $\boldsymbol{O}$ arbitrary (possibly even signaling) boxes. By construction, it clearly holds that

$$
\operatorname{LOSR} \subset \mathrm{GW} .
$$

Furthermore, for any two arbitrary behaviors $\boldsymbol{P}_{0}$ and $\boldsymbol{P}_{\mathrm{f}}$ there exists $\mathcal{W}_{\mathrm{GW}} \in \mathrm{GW}$ such that $\boldsymbol{P}_{\mathrm{f}}=\mathcal{W}_{\mathrm{GW}}\left(\boldsymbol{P}_{0}\right)$. A simple way to see this is by constructing a global wiring $\mathcal{W}_{\mathrm{GW}}$ that bypasses $\boldsymbol{P}_{0}$ and directly generates $\boldsymbol{P}_{\mathrm{f}}$, i.e., by taking $\boldsymbol{O}(\cdot, \cdot \mid \alpha, \beta, \chi, \psi, \cdot, \cdot)=\boldsymbol{P}_{\mathrm{f}}$ for all $\alpha, \beta, \chi$, and $\psi$. Clearly, such $\mathcal{W}_{\text {GW }}$ can map initial local boxes to arbitrary (no-signaling as well as signaling) final boxes. However, as we see in Sec. IV B, surprisingly, this does not imply that GW contains all physical wirings.

Finally, since GW wirings can map no-signaling behaviors out of NS, and since WPICC wirings are well-defined only on behaviors in NS, GW wirings cannot in general be composed with WPICC wirings. This suggests considering a fourth class: the no-signaling wirings, which we denote by NSW. This class comprises all global wirings for which $\boldsymbol{I}, \boldsymbol{O} \in \mathrm{NS}$, i.e., it is defined also by Eq. (10) but with the restriction that the boxes with which the inputs and outputs of the initial behavior are wired are described by no-signaling distributions. The class NSW is highly relevant in a variety of physical scenarios. Nevertheless, below, we prove our results directly for the superset GW. The validity of our results for the subset NSW is automatic by inclusion.

\section{PRIOR CLASSICAL COMMUNICATION IMPROVES BOX DISTINGUISHABILITY}

In this section, we study the inclusion relationships between the different classes of wirings. We show that the set WPICC is strictly larger than LOSR. Even more surprisingly, we also show that WPICC is not contained in GW. Far from being a mere mathematical curiosity, we show the implications of these inclusions in the operational task of distinguishing black boxes.

\section{A. Relative entropy between behaviors as a measure of their distinguishability}

Consider two arbitrary behaviors with equal alphabets of inputs and outputs: $\boldsymbol{P}$, given by Eq. (1), and $\boldsymbol{P}^{\prime}$, given by

$$
\boldsymbol{P}^{\prime}:=\left\{P^{\prime}(a, b \mid x, y)\right\}_{a, b \in[r], x, y \in[s]} .
$$

Imagine next that we wish to distinguish them by choosing their inputs according to a generic joint probability distribution $\boldsymbol{D}:=\{D(x, y)\}_{x, y \in[s]}$ and then comparing the resulting overall input-output statistics, i.e.,

$$
\boldsymbol{P} \cdot \boldsymbol{D}:=\{P(a, b \mid x, y) D(x, y)\}_{a, b \in[r], x, y \in[s]}
$$

and

$$
\boldsymbol{P}^{\prime} \cdot \boldsymbol{D}:=\left\{P^{\prime}(a, b \mid x, y) D(x, y)\right\}_{a, b \in[r], x, y \in[s]} .
$$

The distinguishability between the two probability distributions can be quantified by the relative entropy (RE) $S$, also known as the Kullback-Leibler divergence [28]. More precisely, for any two distributions $\boldsymbol{Q}:=\{Q(z)\}_{z}$ and $\boldsymbol{Q}^{\prime}:=$ $\left\{Q^{\prime}(z)\right\}_{z}$, the RE of $\boldsymbol{Q}$ with respect to $\boldsymbol{Q}^{\prime}$ is defined as

$$
S\left(\boldsymbol{Q} \| \boldsymbol{Q}^{\prime}\right):=\sum_{z} Q(z) \log \left(\frac{Q(z)}{Q^{\prime}(z)}\right) .
$$

$S$ is the most-widely accepted measure of distinguishability between two probability distributions [28-32]. In the asymptotic infinite-sample scenario [28,30,31], $S\left(\boldsymbol{Q} \| \boldsymbol{Q}^{\prime}\right)$ quantifies the statistical confidence that a sample $z$ generated by $\boldsymbol{Q}$ gives, on average, in favor of the hypothesis that the data have indeed been sampled from $Q$ and against the hypothesis that the data have been produced by $\boldsymbol{Q}^{\prime}$.

Thus the RE

$$
\begin{aligned}
S\left(\boldsymbol{P} \cdot \boldsymbol{D} \| \boldsymbol{P}^{\prime} \cdot \boldsymbol{D}\right) & \\
& :=\sum_{a, b \in[r], x, y \in[s]} P(a, b \mid x, y) D(x, y) \log \left(\frac{P(a, b \mid x, y)}{P^{\prime}(a, b \mid x, y)}\right) \\
& =\sum_{x, y \in[s]} D(x, y) S\left(\boldsymbol{P}(\cdot, \cdot \mid x, y) \| \boldsymbol{P}^{\prime}(\cdot, \cdot \mid x, y)\right)
\end{aligned}
$$

of $\boldsymbol{P} \cdot \boldsymbol{D}$ with respect to $\boldsymbol{P}^{\prime} \cdot \boldsymbol{D}$ measures the average distinguishability between the outputs of $\boldsymbol{P}$ and $\boldsymbol{P}^{\prime}$ when the inputs are chosen according to the common distribution D. In Eq. (15), $\boldsymbol{P}(\cdot, \cdot \mid x, y)$ and $\boldsymbol{P}^{\prime}(\cdot, \cdot \mid x, y)$ stand for the probability distributions over the outputs obtained from $\boldsymbol{P}$ and $\boldsymbol{P}^{\prime}$, respectively, for a fixed choice of inputs $(x, y)$.

This motivates a definition for the relative entropy between behaviors, namely, by optimizing the overall distinguishability $S\left(\boldsymbol{P} \cdot \boldsymbol{D} \| \boldsymbol{P}^{\prime} \cdot \boldsymbol{D}\right)$ over all possible input distributions $\boldsymbol{D}$. 
Definition 1 (behavior RE). The relative entropy $S_{\mathrm{b}}\left(\boldsymbol{P} \| \boldsymbol{P}^{\prime}\right)$ of $\boldsymbol{P}$ with respect to $\boldsymbol{P}^{\prime}$ is defined by

$$
\begin{aligned}
S_{\mathrm{b}}\left(\boldsymbol{P} \| \boldsymbol{P}^{\prime}\right) & :=\max _{\boldsymbol{D}} S\left(\boldsymbol{P} \cdot \boldsymbol{D} \| \boldsymbol{P}^{\prime} \cdot \boldsymbol{D}\right) \\
& =\max _{x, y \in[s]} S\left(\boldsymbol{P}(\cdot, \cdot \mid x, y) \| \boldsymbol{P}^{\prime}(\cdot, \cdot \mid x, y)\right) .
\end{aligned}
$$

Equality (16) follows immediately from Eq. (15) and the positive semidefiniteness of $S\left(\boldsymbol{P}(\cdot, \cdot \mid x, y) \| \boldsymbol{P}^{\prime}(\cdot, \cdot \mid x, y)\right)$. $S_{\mathrm{b}}\left(\boldsymbol{P} \| \boldsymbol{P}^{\prime}\right)$ thus measures the statistical distinguishability between the outpu ts of the behaviors $\boldsymbol{P}$ and $\boldsymbol{P}^{\prime}$ when their inputs are chosen probabilistically according to the optimal probability distribution $\boldsymbol{D}$, but this reduces to their statistical distinguishability when the inputs are deterministically fixed at their optimal combination of values, i.e., at the values $(x, y)$ that maximize the output distinguishability in question.

In turn, $S_{b}$ admits an operational interpretation in terms of hypothesis testing. Consider a pair of black-box measurement devices with two possible behaviors, $\boldsymbol{P}$ or $\boldsymbol{P}^{\prime}$, whose inputs are chosen according to a known probability distribution $\boldsymbol{D}$. Imagine further that one suspects that the box is described by $\boldsymbol{P}$ and one would like to rule out the possibility that it is described by $\boldsymbol{P}^{\prime}$ by analyzing only the produced output data. Then, $S_{\mathrm{b}}\left(\boldsymbol{P} \| \boldsymbol{P}^{\prime}\right)$ measures the statistical confidence that an average sample from the output-input distribution $\boldsymbol{P} \cdot \boldsymbol{D}$ gives in favor of the hypothesis that it was indeed output by $\boldsymbol{P} \cdot \boldsymbol{D}$ and against the hypothesis that it was produced by $\boldsymbol{P}^{\prime} \cdot \boldsymbol{D}$, for the best choice of input input distributions $\boldsymbol{D}$ [24]. That is, the higher the value of $S_{\mathrm{b}}\left(\boldsymbol{P} \| \boldsymbol{P}^{\prime}\right)$ is, the smaller the probability is that one mistakes average samples from $\boldsymbol{P} \cdot \boldsymbol{D}$ for samples from $\boldsymbol{P}^{\prime} \cdot \boldsymbol{D}$. The optimal choice of $\boldsymbol{D}$ requires, clearly, full knowledge of $\boldsymbol{P}$ and $\boldsymbol{P}^{\prime}$. However, once $\boldsymbol{D}$ is fixed, the input of every user is chosen solely according to $D$, in particular, independently on the outputs obtained by other users. For more general behavior-distinguishing strategies where the inputs of some users are chosen conditioned on the outputs obtained by others the operational interpretation no longer holds. This will be relevant for the discussion of Sec. IV B.

Finally, the RE between probability distributions is known to be contractive-i.e., nonincreasing-under all linear maps between probability distributions. Contractivity under physical transformations is a property of uttermost importance for any reasonable measure of distinguishability. For instance, the RE between quantum states is known to be contractive under generic completely positive maps [33,34], whereas the RE between steering assemblages is known to be contractive under the free operations of steering [20]. In Appendix B, we prove the analogous for the behavior RE under GW wirings.

Theorem 2 (Contractivity of $S_{\mathrm{b}}$ under GW). Let $\boldsymbol{P}, \boldsymbol{P}^{\prime} \in$ $\mathrm{NS}$ be any two no-signaling behaviors. Then

$$
S_{\mathrm{b}}\left(\mathcal{W}_{\mathrm{GW}}(\boldsymbol{P}) \| \mathcal{W}_{\mathrm{GW}}\left(\boldsymbol{P}^{\prime}\right)\right) \leqslant S_{\mathrm{b}}\left(\boldsymbol{P} \| \boldsymbol{P}^{\prime}\right)
$$

for all $\mathcal{W}_{\mathrm{GW}} \in \mathrm{GW}$.

Note that, by the inclusion relationship (11), Theorem 2 automatically implies that $S_{\mathrm{b}}$ is contractive under LOSR wirings. Consequently, if only LOSR or GW wirings are considered, $S_{\mathrm{b}}$ can be taken as a physically reasonable measure of distinguishability between behaviors. In the next section, we show that, surprisingly, this does not hold for WPICC wirings.

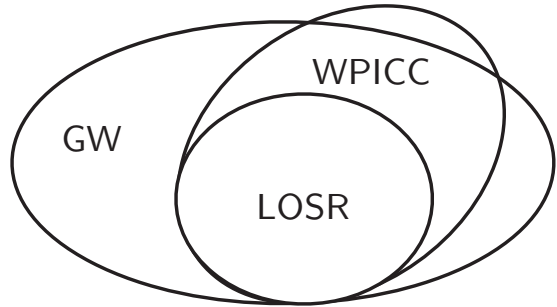

FIG. 2. Inclusion relationships among the three classes of wirings. In spite of being nonlocality free, some wirings with prior-to-input classical communication are out of the set of global wirings, which are, in general, not nonlocality free.

\section{B. WPICC outperforms GW at distinguishing behaviors}

The RE between behaviors proves additionally a useful tool to assess the relationship between the different classes of wirings. From the study of $S_{\mathrm{b}}$, we discover an unexpected inequivalence between WPICC and GW. For that, we first realize the following surprising fact.

Theorem 3 (Noncontractivity of $S_{\mathrm{b}}$ under WPICC). There exist wirings $\mathcal{W}_{\text {WPICC }} \in$ WPICC and behaviors $\boldsymbol{P}, \boldsymbol{P}^{\prime} \in$ NS such that

$$
S_{\mathrm{b}}\left(\mathcal{W}_{\text {WPICC }}(\boldsymbol{P}) \| \mathcal{W}_{\text {WPICC }}\left(\boldsymbol{P}^{\prime}\right)\right)>S_{\mathrm{b}}\left(\boldsymbol{P} \| \boldsymbol{P}^{\prime}\right) .
$$

The theorem is proven in Appendix $\mathrm{C}$ by explicit example construction. An immediate implication is that the class WPICC is not only not equivalent to LOSR but also it is not even contained in GW. This follows as a corollary of Theorem 3 together with Theorem 2.

Corollary 4 (Noninclusion of WPICC in GW). There exist wirings $\mathcal{W}_{\text {WPICC }} \in$ WPICC such that $\mathcal{W}_{\text {WPICC }} \notin \mathrm{GW}$. That is,

$$
\text { WPICC } \not \subset \text { GW. }
$$

The corollary reveals a very unexpected feature of the internal geometry of the set of wirings, schematically depicted in Fig. 2.

Before we finish this section, let us shortly elaborate on the physical implications of Theorem 3 in the informationtheoretic task of distinguishing a behavior $\boldsymbol{P}$ from another $\boldsymbol{P}^{\prime}$ mentioned above. Theorem implies that it is sometimes better to first apply a WPICC wiring $\mathcal{W}_{\text {WPICC }}$ to the black-box pair, and only then sample the outputs to analyze, than to directly sample the outputs from the unprocessed boxes. The optimal choice of inputs then depends on $\mathcal{W}_{\text {WPICC }}(\boldsymbol{P})$ and $\mathcal{W}_{\text {WPICC }}\left(\boldsymbol{P}^{\prime}\right)$ instead of $\boldsymbol{P}$ and $\boldsymbol{P}^{\prime}$. From the theorem, one clearly concludes that WPICC provides an advantage over LOSR, and even GW, as for what the possible preprocessing strategies of the boxes concerns. Nevertheless, Theorem 3 also tells us, on the other hand, that $S_{\mathrm{b}}$, as defined in Definition 1, cannot be considered a satisfactory measure of the total distinguishability of $\boldsymbol{P}$ from $\boldsymbol{P}^{\prime}$. The reason for this is that a basic requirement for a bona fide measure of total distinguishability between two objects is that it does not increase under physical transformations on the objects, which, by virtue of Theorem 3 , is not fulfilled by $S_{\mathrm{b}}$. This may at first sight seem contradictory with the operational interpretation that we provided for $S_{\mathrm{b}}\left(\boldsymbol{P} \| \boldsymbol{P}^{\prime}\right)$ above, right after Definition 1. However, the key point is that, there, the inputs are 
chosen solely according to $D$, whereas here the input choice of one part can depend on the outputs of another part. That is, $S_{\mathrm{b}}\left(\boldsymbol{P} \| \boldsymbol{P}^{\prime}\right)$ quantifies the average distinguishability of $\boldsymbol{P}$ from $\boldsymbol{P}^{\prime}$ in scenarios where the pair of boxes is treated as a monopartite object where all the inputs are chosen before any output is generated. A satisfactory definition of the total distinguishability of $\boldsymbol{P}$ from $\boldsymbol{P}^{\prime}$ should involve an optimization not just over the input probability distributions, as in Eq. (16), but actually over all possible WPICC wirings, which exploit the bipartite nature of the pair by allowing the input of one user to depend on the output of the other one. We leave such formal definition for future work.

The previous discussion is particularly relevant in scenarios where one wishes to estimate the statistical confidence that nonlocal behaviors give as nonlocality proofs [24], i.e., the average distinguishability between a given nonlocal behavior and any behavior in $L$. In the literature, $S_{\mathrm{b}}$ has been employed as the canonical measure of that statistical strength. Nonetheless, the analysis above puts the canonical approach into question and suggests studying distinguishability measures from a resource-theoretic perspective. This is what we do in the next two sections.

\section{BELL NONLOCALITY MONOTONES}

The basic necessary condition for a function to be a satisfactory measure of Bell nonlocality from a resourcetheoretic viewpoint is that it is nonincreasing under the free operations for Bell nonlocality [15,17,18]. For the free wirings, this is formalized by the following definition.

Definition 5 (LOSR and WPICC monotones). A function $f: \mathrm{NS} \rightarrow \mathbb{R}_{\geqslant 0}$ is an LOSR(WPICC) monotone if (i) $f(\boldsymbol{P})=$ 0 for all $\boldsymbol{P} \in \mathrm{L}$ and (ii) for any $\boldsymbol{P} \in \mathrm{NS}, f(\boldsymbol{P}) \geqslant f(\mathcal{W}(\boldsymbol{P}))$ for all $\mathcal{W} \in$ LOSR (WPICC).

Note that, due to the inclusion relation (9), any WPICC monotone is automatically also an LOSR monotone. In the following lemma we show that the converse implication also holds.

Lemma 6 (Bell nonlocality monotones). Let $\quad f: \mathrm{NS} \rightarrow$ $\mathbb{R}_{\geqslant 0}$ be an LOSR monotone; then $f$ is also a WPICC monotone.

The lemma follows from Eq. (8) and from the fact that any convex mixture of a given $\boldsymbol{P}$ with a local behavior (even when the latter is a function of $\boldsymbol{P}$ ) can always be realized by some specific LOSR wiring applied on $\boldsymbol{P}$ [17]. This implies that for every $\mathcal{W} \in$ WPICC and $\boldsymbol{P} \in \mathrm{NS}$, there exists some $\mathcal{W}^{\prime} \in$ LOSR such that $\mathcal{W}(\boldsymbol{P})=\mathcal{W}^{\prime}(\boldsymbol{P})$. Hence it follows that

$$
f(\mathcal{W}(\boldsymbol{P}))=f\left(\mathcal{W}^{\prime}(\boldsymbol{P})\right) \leqslant f(P)
$$

where the inequality is due to the LOSR monotonicity of $f$. The lemma motivates a unified definition of quantifiers of Bell nonlocality.

Definition 7 (Bell nonlocality monotones). We call any LOSR or WPICC monotone a Bell nonlocality monotone.

Next, we construct a Bell nonlocality monotone based on the behavior RE of Definition 1.

\section{Relative entropy of nonlocality}

One can define the relative entropy of Bell nonlocality following the analogous procedure to that used for the REs of entanglement [35] or steering [20], for example. That is, for any $\boldsymbol{P} \in \mathrm{NS}$, the RE of nonlocality of $\boldsymbol{P}$ is defined as

$$
S_{\mathrm{nl}}(\boldsymbol{P}):=\min _{\boldsymbol{P}_{\mathrm{L}} \in \mathrm{L}} S_{\mathrm{b}}\left(\boldsymbol{P} \| \boldsymbol{P}_{\mathrm{L}}\right) .
$$

In other words, $S_{\mathrm{nl}}(\boldsymbol{P})$ is the minimal behavior RE of $\boldsymbol{P}$ with respect to any local behavior. The RE (21) was originally introduced in Ref. [24] as one of the variants of the so-called statistical strength of nonlocality proofs, using game-theoretic considerations, as discussed in detail in Sec. VI. An advantage of defining the RE of nonlocality in terms of the RE between behaviors as in Eq. (16) is that one can prove that $S_{\mathrm{nl}}$ is a resource-theoretically valid measure of Bell nonlocality with the toolbox developed originally for the REs of entanglement [35] or steering [20]. Furthermore, with the same toolbox one can also show convexity of $S_{\mathrm{nl}}$, which is another convenient property for a quantifier of any form of quantum resources. All this is formalized with the following.

Theorem 8 (Bell monotonicity and convexity of $S_{\mathrm{nl}}$ ). The RE of Bell nonlocality $S_{\mathrm{nl}}$ is a Bell nonlocality monotone. Moreover, it is also convex, i.e.,

$$
S_{\mathrm{nl}}\left(\mu \boldsymbol{P}+(1-\mu) \boldsymbol{P}^{\prime}\right) \leqslant \mu S_{\mathrm{nl}}(\boldsymbol{P})+(1-\mu) S_{\mathrm{nl}}\left(\boldsymbol{P}^{\prime}\right),
$$

for all $0 \leqslant \mu \leqslant 1$ and all $\boldsymbol{P}, \boldsymbol{P}^{\prime} \in \mathrm{NS}$.

The proof of the theorem is given in Appendix D.

\section{CONNECTION TO THE STATISTICAL STRENGTH OF NONLOCALITY PROOFS}

In Ref. [24], van Dam, Grunwald, and Gill (vDGG) introduced an information-theoretic measure of the statistical strength of nonlocal behaviors as Bell nonlocality proofs, named the statistical strength of nonlocality proofs. For any nonlocal behavior $\boldsymbol{P}_{\mathrm{NL}} \in \mathrm{NS}$, this measure quantifies the minimum statistical confidence that an output sample generated by $\boldsymbol{P}_{\mathrm{NL}}$ gives in support of the hypothesis HNL that the outputs have indeed been generated from $\boldsymbol{P}_{\mathrm{NL}}$ and against the hypothesis HL that the data have been produced by any behavior $\boldsymbol{P}_{\mathrm{L}} \in \mathrm{L}$, when the inputs are chosen according to a probability distribution $\boldsymbol{D}$, and maximising over $\boldsymbol{D}$. vDGG proposed three variants, given by three different constraints on the allowed input distribution $\boldsymbol{D}$ :

$$
\begin{aligned}
S_{\mathrm{u}}\left(\boldsymbol{P}_{\mathrm{NL}}\right) & :=\min _{\boldsymbol{P}_{\mathrm{L}} \in \mathrm{L}} S\left(\boldsymbol{P}_{\mathrm{NL}} \cdot \boldsymbol{D}^{(u)} \| \boldsymbol{P}_{\mathrm{L}} \cdot \boldsymbol{D}^{(u)}\right), \\
S_{\mathrm{uc}}\left(\boldsymbol{P}_{\mathrm{NL}}\right) & :=\max _{\boldsymbol{D} \in \mathrm{UC}} \min _{\boldsymbol{P}_{\mathrm{L}} \in \mathrm{L}} S\left(\boldsymbol{P}_{\mathrm{NL}} \cdot \boldsymbol{D} \| \boldsymbol{P}_{\mathrm{L}} \cdot \boldsymbol{D}\right), \\
S_{\mathrm{c}}\left(\boldsymbol{P}_{\mathrm{NL}}\right) & :=\max _{\boldsymbol{D}} \min _{\boldsymbol{P}_{\mathrm{L}} \in \mathrm{L}} S\left(\boldsymbol{P}_{\mathrm{NL}} \cdot \boldsymbol{D} \| \boldsymbol{P}_{\mathrm{L}} \cdot \boldsymbol{D}\right) .
\end{aligned}
$$

The labels u, uc, and c, stand respectively for uniform, uncorrelated, and correlated. $\boldsymbol{D}^{(u)}$ is the uniform probability distribution of settings, with elements $I_{\mathrm{u}}(x, y)=1 / s^{2}$ for all $x, y \in[s]$. The maximization in Eq. (23b) is restricted to the subset UC:= $\left.\boldsymbol{D}: \boldsymbol{D}=\boldsymbol{D}_{X} \cdot \boldsymbol{D}_{Y}\right\}$ of all probability distributions of uncorrelated settings chosen independently with arbitrary local distributions $\boldsymbol{D}_{X}$ and $\boldsymbol{D}_{Y}$. In contrast, the 
maximization in Eq. (23c) runs over the whole the simplex of all probability distributions $\boldsymbol{D}$, including those for which the inputs are correlated.

We note, also, that, in Ref. [18], measures analogous to (23a) and (23c) have been considered for contextuality.

\section{A. Interpretation of the three variants of the strength of nonlocality proofs}

vDGG interpret the three variants in Eq. (23) in terms of a two-player game. One of the players, QUANTUM, supporter of the hypothesis HNL, wants to convince the other one, CLASSICAL, supporter of HL, that HNL is true and HL is false. To this end, QUANTUM takes a box with nonlocal behavior $\boldsymbol{P}_{\mathrm{NL}} \notin \mathrm{L}$, chooses its inputs according to a distribution $\boldsymbol{D}$, and samples outputs from it. The more distinguishable the resulting input-output distribution is from any one generated by a local behavior $\boldsymbol{P}_{\mathrm{L}} \in \mathrm{L}$, for the same input distribution $\boldsymbol{D}$, the more evident it becomes for CLASSICAL that QUANTUM is right. In turn, each of the variants in Eq. (23) measures the optimal (over $\boldsymbol{D}$ ) asymptotic statistical confidence in HNL when QUANTUM is allowed to choose the inputs uniformly, uncorrelated, or arbitrarily. Of all three definitions, vDGG favor Eq. (23b), corresponding to uncorrected inputs, as the most reasonable one. On the one hand, the authors see no physical reason why QUANTUM should restrict to uniformly chosen inputs to rightfully convince CLASSICAL. On the other one, they argue that using generic (possibly correlated) inputs makes QUANTUM's case weaker, as it could give CLASSICAL the impression that some hidden communication between Alice and Bob might be taking place.

While it is certainly true that choosing inputs uniformly is unnecessarily restrictive, we find the restriction to uncorrelated inputs unnecessary too. As is well known, correlations between Alice and Bob's inputs cannot be used by QUANTUM to fake nonlocality using a local behavior (see, e.g., Ref. [36]). What could, in contrast, be used to cheat CLASSICAL are correlations between the inputs and the hidden variable [37]. However, such correlations are totally independent of whether the inputs are uniform, uncorrelated, or correlated. Thus we see no reason why QUANTUM should restrict to independent inputs to rightfully convince CLASSICAL. As for what game-theoretic interpretation concerns, we view Eq. (23c), corresponding to generic inputs, as the most reasonable definition of all three.

On the other hand, from a resource-theoretic perspective, QUANTUM's point should be made based on nonlocality measures in the sense of Definitions 5 and 7. That is, the statistical strength of nonlocality proofs should be quantified by a Bell nonlocality monotone. Otherwise, one may run into situations where the statistical strength is ill-defined. To see this, suppose that QUANTUM takes, as nonlocality proof, a given nonlocal behavior $\boldsymbol{P}_{\mathrm{NL}} \notin \mathrm{L}$. According to Eq. (23), its statistical strength of nonlocality should be $S_{\mathrm{i}}\left(\boldsymbol{P}_{\mathrm{NL}}\right)$, where the subindex i can represent any of three input-choice strategies. However, before generating the outputs, QUANTUM has the freedom of modifying his nonlocality proof by having Alice and Bob apply a free wiring $\mathcal{W}$ to it, for instance $\mathcal{W} \in \operatorname{LOSR}$ or $\mathcal{W} \in$ WPICC. CLASSICAL cannot complain about this, since, by definition, $\mathcal{W}$ cannot create nonlocality, but the statistical strength would then change from $S_{\mathrm{i}}\left(\boldsymbol{P}_{\mathrm{NL}}\right)$ to $S_{\mathrm{i}}\left(\mathcal{W}\left(\boldsymbol{P}_{\mathrm{NL}}\right)\right)$, which may be greater than $S_{\mathrm{i}}\left(\boldsymbol{P}_{\mathrm{NL}}\right)$. Furthermore, this process could be repeated indefinitely, obtaining every time, according to Eq. (23), a different (wiring-dependent) value of the statistical strength. This problem is circumvented if the statistical strength is defined so as to satisfy nonlocality monotonicity.

\section{B. Monotonicity and nonmonotonicity of the strengths of nonlocality}

We next study whether the three variants of the strength of nonlocality proofs given by Eq. (23) are Bell nonlocality monotones. First of all, note that [24]

$$
S_{\mathrm{nl}}=S_{\mathrm{c}}
$$

(see Appendix E for an explicit proof). Hence the monotonicity of $S_{\mathrm{c}}$ follows automatically from Theorem 8. On the other hand, for $S_{\mathrm{u}}$ and $S_{\mathrm{uc}}$, monotonicity is addressed by the following two lemmas, which we prove in Appendixes $F$ and $\mathrm{G}$.

Theorem 9 (Nonmonotonicity of $S_{u}$ ). $S_{\mathrm{u}}$ is not an LOSR monotone. Hence it is, in addition, neither a WPICC monotone nor a Bell nonlocality monotone.

Theorem 10 (Monotonicity of $S_{u c}$ ). $S_{\mathrm{uc}}$ is a Bell nonlocality monotone.

The fact that $S_{\mathrm{u}}$ is not monotone under free wirings rules it out as a resource-theoretic consistent candidate for the statistical strength of nonlocality. In contrast, $S_{\text {uc }}$ is consistent with both resource theories of Bell nonlocality, the one based on LOSR as well as that based on WPICC. Nonetheless, it is worth mentioning that our proof of Theorem 10 directly relies on the fact that the hypothesis HL against one is testing is that $\boldsymbol{P}_{\mathrm{NL}} \notin \mathrm{L}$ (see Appendix G) and does not work against stronger hypotheses. For instance, imagine the hypothetical situation of a third player, POSTQUANTUM, supporter of the hypothesis HPQ that the data have been generated by a postquantum no-signaling behavior $\boldsymbol{P}_{\mathrm{PQ}} \in \mathrm{NS} \backslash \mathrm{Q}$ and against the hypothesis HQ that they have been produced by any quantum behavior $\boldsymbol{P}_{\mathrm{Q}} \in \mathrm{Q}$. POSTQUANTUM wants to convince QUANTUM that quantum theory is violated and offers $\boldsymbol{P}_{\mathrm{PQ}}$ as a postquantumness proof. The analogous of Eq. (23b) relevant for such a scenario would then be

$$
S_{\mathrm{uc}}^{(\mathrm{Q})}\left(\boldsymbol{P}_{\mathrm{PQ}}\right):=\max _{\boldsymbol{D} \in \mathrm{UC}} \min _{\boldsymbol{P}_{\mathrm{Q}} \in \mathrm{Q}} S\left(\boldsymbol{P}_{\mathrm{PQ}} \cdot \boldsymbol{D} \| \boldsymbol{P}_{\mathrm{Q}} \cdot \boldsymbol{D}\right) .
$$

Accordingly, monotonicity should be shown under quantum wirings, defined analogous to Eq. (7) but with the inputs and outputs wired to boxes in $Q$ instead of $L$. The proof of monotonicity given in Appendix G (specifically, Lemma 12 there) does not hold for $S_{\mathrm{uc}}^{(\mathrm{Q})}$ under quantum wirings. We leave as an open question whether $S_{\mathrm{uc}}^{(\mathrm{Q})}$ is a monotone under quantum wirings.

\section{DISCUSSION}

We would like to finish with a few relevant remarks about our results and some open questions. First of all, at first blush, Theorem 3 (our central theorem), which proves nonmonotonicity of the behavior relative entropy $S_{\mathrm{nl}}$ under WPICC wirings, may give the impression that it could be 
possible to increase the distinguishability of a given nonlocal behavior from the local ones by a WPICC wiring. This would be directly relevant, e.g., for nonlocality certification. However, we know from Theorem 8, which proves WPICC monotonicity of the relative entropy of nonlocality $S_{\mathrm{nl}}$, that such an increase is impossible. Interestingly, WPICC wirings manage to increase $S_{\mathrm{b}}$ but seemingly within the subspac e of zero $S_{\mathrm{nl}}$. In fact, the two exemplary behaviors $\boldsymbol{P}$ and $\boldsymbol{P}^{\prime}$ given in the proof of Theorem 3 , whose relative entropy increases under a WPICC wiring, are both local, i.e., both have zero $S_{\mathrm{nl}}$. While we have not seriously attempted to find nonlocal behaviors, or a local and a nonlocal one, for which $S_{\mathrm{b}}$ increases under WPICC, we do not rule out that possibility. A possible search strategy for such examples could be to consider convex combinations of the exemplary behaviors of the proof of Theorem 3, and similar WPICC wirings too, and numerically optimize the measurement settings to assess $S_{\mathrm{b}}$.

Second, one may wonder about the quantitative sustainment of Theorem 3. We have phrased the theorem in qualitative terms, focusing on the fact that the relative entropy can increase and without regard of how much the increase can be. However, the concrete examples that we present in the proof of the theorem, in Appendix C, show that $S_{\mathrm{b}}$ can be doubled, for the specific case of at most two inputs and two outputs per user, and that it can even increase unboundedly with the size of the input-output alphabets in the general case, remarkably.

Third, as already mentioned, another consequence of Theorem 3 is that a bona fide measure of the overall distinguishability between behaviors must explicitly take into account that behaviors are multipartite objects for which some of the inputs can be chosen depending on the outputs of other users.

Fourth, concerning the strength of nonlocality proofs, operational consistency demands that its definition incorporates Bell nonlocality monotonicity as a built-in property. We have shown that the variant for which the inputs are sampled from the uniform distribution does not fulfill this. The other two variants introduced in Ref. [24], with correlated and uncorrelated inputs, are both Bell nonlocality monotones, although (as an interesting side remark) it is an open question whether the variant with uncorrelated inputs would be consistent with an operational framework with quantum wirings. Either way, from the latter two variants, the fully general one allowing for correlated inputs seems the most appropriate to us. On the one hand, we find the restriction to uncorrelated inputs unnecessary and, on the other one, the variant with correlated inputs coincides with the $S_{\mathrm{nl}}$ as defined directly in terms of $S_{\mathrm{b}}$.

Fifth, as we have discussed, since $L$ is closed under both LOSR and WPICC, from the mathematical point of view, both classes can be taken as valid free operations for Bell nonlocality. However, from the physical viewpoint, one class could be more appropriate than the other in some situations. This depends on the natural physical constraints native of the specific task for which the nonlocal correlations are serving as resource. For instance, since WPICC allows for communication about inputs and outputs of the initial box, it is legitimate to ask wether WPICC can indeed be allowed in, say, quantum-key distribution. There, depending on the protocol, security constraints might impose LOSR over WPICC as the adequate class of harmless operations.
In conclusion, from a fundamental perspective, our work reveals unexpected features of the internal geometry of the set of wirings and characterizes the connections between the two resource-theoretic paradigms for Bell nonlocality. From an applied one, in turn, our findings may be relevant to the operational task of distinguishing black-box measurement devices from a restricted set of measurement settings. This may for instance be the case in nonlocality certification within cryptographic protocols. There, one may be interested in certifying a nonlocal target behavior from the same measurements used in the protocol, so that a potential eavesdropper does not know which experimental runs are used for the protocol itself and which ones for the certification.

\section{ACKNOWLEDGMENTS}

We thank J. I. de Vicente for useful discussions and for bringing Lemma 6 to our attention. R.G. acknowledges funding from DFG (Grant No. GA 2184/2-1) and ERC (TAQ). L.A. acknowledges financial support from the Brazilian agencies CNPq, FAPERJ, CAPES, and INCT-IQ.

\section{APPENDIX A: PARAMETRIZATION OF THE CLASS WPICC}

Here we derive the explicit analytic parametrization of a generic wiring $\mathcal{W}_{\text {WPICC }} \in$ WPICC, whose simplified form is given in Eq. (8). To this end, we use a general decision tree. During the preparation phase, the experimenters decide, using shared randomness, if Alice measures first, if Bob measures first, or if no-one measures. For the first two cases, the user that measures first communicates to the other one his or her chosen input ( $x$ or $y$ ) as well his or her obtained output ( $a$ or $b$ ). Then, conditioned on the two communicated dits, the other user decides whether or not to measure. This gives altogether five different cases, and a generic WPICC wiring allows, of course, for probabilistic mixings of all five branches. The branch where no-one measures during the preparation leads, by definition, to an LOSR overall resulting wiring. For the two branches where both users measure during the preparation (either Alice first and Bob second or vice versa), the users end up in the preparation phase with the correlated random dits $a$, $x, b$, and $y$. One may be tempted to think that the latter two branches also result in an overall LOSR wiring, but this is not the case, as we show next.

Let us first group the five cases into three main branches and analyze the transformations experienced by $\boldsymbol{P}_{0}$, due to both preparation and measurement phases, along each branch as follows.

(i) Both Alice and Bob measure their initial boxes. Suppose Bob measures first. That is, he chooses $y$ according to a single-partite probability distribution $\boldsymbol{D}_{Y}$. His box thus outputs $b$ with probability $P_{0}(b \mid y)$, given by his marginal behavior from $\boldsymbol{P}_{0}$. He sends both dits $y$ and $b$ to Alice. She, in turn, chooses $x$ according to a single-partite probability distribution $\boldsymbol{D}_{X \mid b, y}$, which explicitly depends on $b$ and $y$, and obtains $a$ with conditional probability $P_{0}(a \mid x, b, y)$. In general, Alice can also communicate her dits $x$ and $a$ to Bob, so both parties finish the preparation phase with all four dits. Since the initial inputs are already chosen, the resulting behavior is a joint probability 
distribution $\boldsymbol{P}_{0}^{(B \rightarrow A)}$ with outputs only ( $a, b, x$, and $\left.y\right)$, whose elements are

$$
\begin{aligned}
\boldsymbol{P}_{0}^{(B \rightarrow A)}(a, x, b, y) & =D_{Y}(y) P_{0}(b \mid y) D_{X \mid b, y}(x) P_{0}(a \mid x, b, y) \\
& =D_{Y}(y) P_{0}(a, b \mid x, y) D_{X \mid b, y}(x) .
\end{aligned}
$$

Clearly, since it has no inputs, the resulting behavior $\boldsymbol{P}_{0}^{(B \rightarrow A)}$ has a local-hidden variable model. Finally, in the measurement phase, $\boldsymbol{P}_{0}^{(B \rightarrow A)}$ undergoes the most generic LOSR wiring acting on behaviors without inputs and for which both users know each other's initial dits. This is explicitly parametrized by $\boldsymbol{P}_{\mathrm{f}}^{(B \rightarrow A)}=\mathcal{W}_{\mathrm{LOSR}}^{(B \rightarrow A)}\left(\boldsymbol{P}_{0}^{(B \rightarrow A)}\right)$, with

$$
\begin{aligned}
P_{\mathrm{f}}^{(B \rightarrow A)}(\alpha, \beta \mid \chi, \psi):= & \sum_{a, b, x, y} O_{a, b, x, y}^{(\mathrm{L})}(\alpha, \beta \mid \chi, \psi) \\
& \times P_{0}^{(B \rightarrow A)}(a, b \mid x, y) .
\end{aligned}
$$

For each $a, b \in[r]$ and $x, y \in[s], \boldsymbol{O}_{a, b, x, y}^{(\mathrm{L})}$ is a local behavior (with respect to $\alpha$ and $\beta$ as outputs and $\chi$ and $\psi$ as inputs) that depends arbitrarily on $a, b, x$, and $y$, reflecting the fact that both users know each other's initial dits. Clearly, since $\boldsymbol{P}_{0}^{(B \rightarrow A)} \in \mathrm{L}$, the final behavior $\boldsymbol{P}_{\mathrm{f}}^{(B \rightarrow A)}$ is also in $\mathrm{L}$. To end up with, if Alice measures first, one obtains the final behavior $\boldsymbol{P}_{\mathrm{f}}^{(A \rightarrow B)} \in \mathrm{L}$, defined analogous to $\boldsymbol{P}_{\mathrm{f}}^{(B \rightarrow A)}$.

(ii) Either Alice or Bob measures her or his initial box. Suppose it is Bob who makes the measurement. Precisely, Fig. 1(b) represents an example of this situation. In this case, Bob's actions are the same as in the previous branch. Alice, in contrast, does not measure her device until the measurement phase, but she holds a copy of Bob's dits $b$ and $y$. The resulting behavior $\boldsymbol{P}_{0}^{(B)}$ has a single input ( $x$, on Alice's side) and its elements are

$$
P_{0}^{(B)}(a, b, y \mid x)=D_{Y}(y) P_{0}(a, b \mid x, y) .
$$

Clearly, since only one side has inputs, the resulting behavior $\boldsymbol{P}_{0}^{(B)}$ has a local-hidden variable model too. Finally, in the measurement phase, $\boldsymbol{P}_{0}^{(B)}$ undergoes the most generic LOSR wiring on behaviors with inputs only on Alice's side and for which Alice knows Bob's initial dits. This is parametrized by $\boldsymbol{P}_{\mathrm{f}}^{(B)}=\mathcal{W}_{\mathrm{LOSR}}^{(B)}\left(\boldsymbol{P}_{0}^{(B)}\right)$, with

$$
\begin{aligned}
P_{\mathrm{f}}^{(B)}(\alpha, \beta \mid \chi, \psi):= & \sum_{a, b, x, y} O_{b, y}^{(\mathrm{L})}(\alpha, \beta \mid a, x, \chi, \psi) \\
& \times P_{0}^{(B)}(a, b, y \mid x) I_{b, y}^{(A)}(x \mid \chi) .
\end{aligned}
$$

For each $b \in[r]$ and $y \in[s], \boldsymbol{O}_{b, y}^{(\mathrm{L})}$ is a local behavior (with respect to $\alpha$ and $\beta$ as outputs and $a, x, \chi$, and $\psi$ as inputs) and $\boldsymbol{I}_{b, y}^{(A)}$ is a single-partite behavior. Both depend arbitrarily on $b$ and $y$, reflecting the fact that Alice knows Bob's initial dits. Clearly, since $\boldsymbol{P}_{0}^{(B)} \in \mathrm{L}$, the final behavior $\boldsymbol{P}_{\mathrm{f}}^{(B)}$ is also in $\mathrm{L}$. To end up with, if it is instead Alice who measures, one obtains the final behavior $\boldsymbol{P}_{\mathrm{f}}^{(A)} \in \mathrm{L}$, defined analogously to $\boldsymbol{P}_{\mathrm{f}}^{(B)}$.

(iii) None of the parties measures in the preparation phase. In this case, both parties apply directly an LOSR wiring, leading to the final behavior $\boldsymbol{P}_{\mathrm{f}}=\mathcal{W}_{\mathrm{LOSR}}\left(\boldsymbol{P}_{0}\right)$, with $\mathcal{W}_{\mathrm{LOSR}}^{(\text {none }}$ a generic wiring in LOSR.

As mentioned above, probabilistic mixtures of all five cases are admitted in general, leading to the final expression

$$
\begin{aligned}
\mathcal{W}_{\text {WPICC }}\left(\boldsymbol{P}_{0}\right)= & p_{A \rightarrow B} \mathcal{W}_{\mathrm{LOSR}}^{(A \rightarrow B)}\left(\boldsymbol{P}_{0}^{(A \rightarrow B)}\right) \\
& +p_{B \rightarrow A} \mathcal{W}_{\mathrm{LOSR}}^{(B \rightarrow A)}\left(\boldsymbol{P}_{0}^{(B \rightarrow A)}\right) \\
& +p_{A} \mathcal{W}_{\mathrm{LOSR}}^{A}\left(\boldsymbol{P}_{0}^{A}\right)+p_{B} \mathcal{W}_{\mathrm{LOSR}}^{B}\left(\boldsymbol{P}_{0}^{B}\right) \\
& +p_{\text {None }} \mathcal{W}^{(\text {none })}\left(\boldsymbol{P}_{0}\right)
\end{aligned}
$$

with $p_{A \rightarrow B}, p_{B \rightarrow A}, p_{A}, p_{B}, p_{\text {none }} \geqslant 0$ and $p_{A \rightarrow B}+p_{B \rightarrow A}+$ $p_{A}+p_{B}+p_{\text {none }}=1$. Making, next, in Eq. (A5), the identifications $p:=1-p^{(\text {none })}$ and

$$
\begin{aligned}
L\left(\boldsymbol{P}_{0}\right):= & \frac{1}{p}\left[p_{A \rightarrow B} \mathcal{W}_{\mathrm{LOSR}}^{(A \rightarrow B)}\left(\boldsymbol{P}_{0}^{(A \rightarrow B)}\right)\right. \\
& +p_{B \rightarrow A} \mathcal{W}_{\mathrm{LOSR}}^{(B \rightarrow A)}\left(\boldsymbol{P}_{0}^{(B \rightarrow A)}\right) \\
& \left.+p_{A} \mathcal{W}_{\mathrm{LOSR}}^{A}\left(\boldsymbol{P}_{0}^{A}\right)+p_{B} \mathcal{W}_{\mathrm{LOSR}}^{B}\left(\boldsymbol{P}_{0}^{B}\right)\right],
\end{aligned}
$$

one arrives at Eq. (8). Note that $L\left(\boldsymbol{P}_{0}\right) \in \mathrm{L}$ for all $\boldsymbol{P}_{0} \in \mathrm{NS}$, because each term in Eq. (A6) is a behavior in $L$.

\section{APPENDIX B: PROOF OF THEOREM 2}

Note first that all wirings $\mathcal{W}_{\mathrm{GW}} \in \mathrm{GW}$ treat the bipartite boxes $\boldsymbol{P}$ and $\boldsymbol{P}^{\prime}$ as if they were monopartite boxes with singlepartite inputs $i=(x, y)$ and output $o=(a, b)$. Similarly, we also make the identifications $\phi=(\chi, \psi)$ and $\gamma=(\alpha, \beta)$. With this notation, we must show that Eq. (17) holds using that $\boldsymbol{P}_{\mathrm{f}}=\mathcal{W}_{\mathrm{GW}}\left(\boldsymbol{P}_{0}\right)$, with

$$
P_{\mathrm{f}}(\gamma \mid \phi):=\sum_{o, i} O(\gamma \mid o, i, \phi) P_{0}(o \mid i) I(i \mid \phi) .
$$

Using Eqs. (14) and (16), we write

$$
\begin{aligned}
S_{\mathrm{b}}\left(\mathcal{W}_{\mathrm{GW}}(\boldsymbol{P}) \mid \mathcal{W}_{\mathrm{GW}}\left(\boldsymbol{P}^{\prime}\right)\right) & =\max _{\phi} \sum_{\gamma} P_{f}(\gamma \mid \phi) \log \left(\frac{P_{f}(\gamma \mid \phi)}{P_{f}^{\prime}(\gamma \mid \phi)}\right) \\
& =\max _{\phi} \sum_{\gamma} \sum_{o, i} O(\gamma \mid o, i, \phi) P_{0}(o \mid i) I(i \mid \phi) \log \left(\frac{\sum_{o, i} O(\gamma \mid o, i, \phi) P_{0}(o \mid i) I(i \mid \phi)}{\sum_{o, i} O(\gamma \mid o, i, \phi) P_{0}^{\prime}(o \mid i) I(i \mid \phi)}\right) \\
& \leqslant \max _{\phi} \sum_{\gamma} \sum_{o, i} O(\gamma \mid o, i, \phi) P_{0}(o \mid i) I(i \mid \phi) \log \left(\frac{O(\gamma \mid o, i, \phi) P_{0}(o \mid i) I(i \mid \phi)}{O(\gamma \mid o, i, \phi) P_{0}^{\prime}(o \mid i) I(i \mid \phi)}\right) \\
& =\max _{\phi} \sum_{\gamma} \sum_{o, i} O(\gamma \mid o, i, \phi) P_{0}(o \mid i) I(i \mid \phi) \log \left(\frac{P_{0}(o \mid i)}{P_{0}^{\prime}(o \mid i)}\right)
\end{aligned}
$$




$$
\begin{aligned}
& =\max _{\phi} \sum_{o, i} P_{0}(o \mid i) I(i \mid \phi) \log \left(\frac{P_{0}(o \mid i)}{P_{0}^{\prime}(o \mid i)}\right) \\
& =\max _{\phi} \sum_{i} I(i \mid \phi) S\left(\boldsymbol{P}(\cdot, i) \| \boldsymbol{P}^{\prime}(\cdot, i)\right) \\
& \leqslant \max _{i} S\left(\boldsymbol{P}(\cdot, i) \| \boldsymbol{P}^{\prime}(\cdot, i)\right) \\
& =S_{\mathrm{b}}\left(\boldsymbol{P} \| \boldsymbol{P}^{\prime}\right),
\end{aligned}
$$

where (B2) follows from (B1), Eq. (B3) from the log sum inequality $\sum_{i} x_{i} \log \left(\sum_{i} x_{i} / \sum_{i} y_{i}\right) \leqslant \sum_{i} x_{i} \log \left(x_{i} / y_{i}\right)$ if $x_{i} \geqslant$ 0 and $y_{i} \geqslant 0 \forall i$, Eq. (B4) from basic algebra, Eq. (B5) from summing over $\gamma$ and using that the probability distributions are normalized, Eq. (B6) from Eq. (14), Eq. (B7) from the fact that the average is smaller than the largest value, and Eq. (B8) from Eq. (16).

\section{APPENDIX C: PROOF OF THEOREM 2}

Our proof strategy consists of constructing two concrete behaviors $\boldsymbol{P}_{0}, \boldsymbol{P}_{0}^{\prime} \in \mathrm{NS}$ and a concrete wiring $\mathcal{W}_{\text {WPICC }} \in$ WPICC such that Eq. (18) is fulfilled. In fact, our construction takes place in the simple scenario of $r_{A}=r_{B}=$ $s_{A}=2$ and $s_{B}=1$. That is, we consider a single input for Bob. Explicitly, $\boldsymbol{P}_{0}=\left\{P_{0}(a, b \mid x)\right\}_{a, b \in[2], x \in[2]}$ and $\boldsymbol{P}_{0}^{\prime}=$

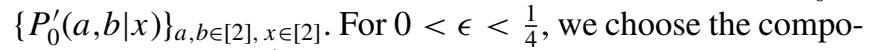
nents of $\boldsymbol{P}_{0}$ and $\boldsymbol{P}_{0}^{\prime}$ as

\begin{tabular}{lcc}
\hline \hline$P_{0}(a, b \mid x)$ & $x=0$ & $x=1$ \\
\hline$a=0, b=0$ & $\frac{1}{2}-\epsilon$ & $\frac{1}{2}-\epsilon$ \\
$a=1, b=0$ & $\epsilon$ & $\epsilon$ \\
$a=0, b=1$ & $\epsilon$ & $\epsilon$ \\
$a=1, b=1$ & $\frac{1}{2}-\epsilon$ & $\frac{1}{2}-\epsilon$ \\
$P_{0}^{\prime}(a, b \mid x)$ & $x=0$ & $x=1$ \\
\hline$a=0, b=0$ & $\epsilon$ & $\frac{1}{2}-\epsilon$ \\
$a=1, b=0$ & $\frac{1}{2}-\epsilon$ & $\epsilon$ \\
$a=0, b=1$ & $\epsilon$ & $\frac{1}{2}-\epsilon$ \\
$a=1, b=1$ & $\frac{1}{2}-\epsilon$ & $\epsilon$ \\
\hline \hline
\end{tabular}

As the reader can immediately verify, both distributions are well normalized. In addition, $P_{0}(b \mid x=0)=P_{0}(b \mid x=1)$ and $P_{0}^{\prime}(b \mid x=0)=P_{0}^{\prime}(b \mid x=1)$, which implies that the behaviors are no signaling. This, together with the fact that Bob has a single input, implies that $\boldsymbol{P}_{0}$ and $\boldsymbol{P}_{0}$ are actually in $\mathrm{L}$.

Let us first give some intuition of why one expects that (18) can hold for these distributions. $S_{\mathrm{b}}\left(\boldsymbol{P}_{0} \| \boldsymbol{P}_{0}^{\prime}\right)=\max _{x} S\left(\boldsymbol{P}_{0}(\cdot, \cdot\right.$ $\left.\mid x) \| \boldsymbol{P}_{0}^{\prime}(\cdot, \cdot \mid x)\right)$ measures the distinguishability between the output distributions resulting from $\boldsymbol{P}_{0}$ and $\boldsymbol{P}_{0}^{\prime}$ when the input is fixed at $x$ maximized over $x$. Note that, due to the fact that $\boldsymbol{P}_{0}$ is independent of $x$ and the symmetries of $\boldsymbol{P}_{0}^{\prime}$, they are equally indistinguishable for $x=0$ and $x=1$. Let us analyze how distinguishable they are for each input value: For $x=0$, when $b=1$ the resulting distributions over $a$ are the same, whereas they are different for $b=0$ (see the first column of the tables). In turn, for $x=1$, when $b=0$ the resulting distributions over $a$ are the same, whereas they are different for $b=1$ (see the second column of the tables). Now, consider the following wiring $\mathcal{W}_{\text {WPICC }} \in$ WPICC: in the preparation phase, Bob presses the only button of his initial box (he has no choice of settings for this, since $s_{b}=1$ ), obtains the bit $b$ as output, and sends it to Alice. In the measurement phase, in turn, Alice receives the input $\chi$ of her final box, ignores it, and chooses the input to her initial box as $x=b$. There are then no further wirings and the final outputs are simply $\alpha=a$ and $\beta=b$. By doing this, Alice and Bob avoid the cases $(x=$ $0, b=1)$ and $(x=1, b=0)$ for which $\boldsymbol{P}_{0}$ and $\boldsymbol{P}_{0}^{\prime}$ behave the same. Heuristically, we expect that this transformation should increase the distinguishability. Let us next prove it rigorously.

First, we rewrite the right-hand side of (18) as

$$
\begin{aligned}
S_{\mathrm{b}}\left(\boldsymbol{P}_{0} \| \boldsymbol{P}_{0}^{\prime}\right) & =\max _{x} S\left(\boldsymbol{P}_{0}(\cdot, \cdot \mid x) \| \boldsymbol{P}_{0}^{\prime}(\cdot, \cdot \mid x)\right) \\
& =S\left(\boldsymbol{P}_{0}(\cdot, \cdot \mid 0) \| \boldsymbol{P}_{0}^{\prime}(\cdot, \cdot \mid 0)\right) \\
& =\sum_{a, b} P_{0}(a, b \mid 0) \log \left(\frac{P_{0}(a, b \mid 0)}{P_{0}^{\prime}(a, b \mid 0)}\right) \\
& =\left(\frac{1}{2}-2 \epsilon\right) \log \left(\frac{\frac{1}{2}-\epsilon}{\epsilon}\right) .
\end{aligned}
$$

The above-mentioned wiring is such that the number of inputs and outputs is preserved, i.e., $r_{A f}=r_{B f}=s_{A f}=2$ and $s_{B f}=1$. The final behaviors are in turn given by $\boldsymbol{P}_{\mathrm{f}}=$ $\mathcal{W}_{\mathrm{GW}}\left(\boldsymbol{P}_{0}\right)$ and $\boldsymbol{P}_{\mathrm{f}}^{\prime}=\mathcal{W}_{\mathrm{GW}}\left(\boldsymbol{P}_{0}^{\prime}\right)$, with

$$
\begin{aligned}
& P_{f}(a, b \mid \chi)=P_{0}(a, b \mid b) \forall a, b, \chi \in[2], \\
& P_{f}^{\prime}(a, b \mid \chi)=P_{0}^{\prime}(a, b \mid b) \forall a, b, \chi \in[2] .
\end{aligned}
$$

We emphasize that both final behaviors are independent of $\chi$, since Alice chooses the input to her initial box as $x=b$ ignoring the value of the final input $\chi$. Explicitly, the resulting components of the final behaviors are given by the following tables:

\begin{tabular}{lcc}
\hline \hline$P_{\mathrm{f}}(a, b \mid \chi)$ & $\chi=0$ & $\chi=1$ \\
\hline$a=0, b=0$ & $\frac{1}{2}-\epsilon$ & $\frac{1}{2}-\epsilon$ \\
$a=1, b=0$ & $\epsilon$ & $\epsilon$ \\
$a=0, b=1$ & $\epsilon$ & $\epsilon$ \\
$a=1, b=1$ & $\frac{1}{2}-\epsilon$ & $\frac{1}{2}-\epsilon$ \\
$P_{\mathrm{f}}^{\prime}(a, b \mid \chi)$ & $\chi=0$ & $\chi=1$ \\
\hline$a=0, b=0$ & $\epsilon$ & $\epsilon$ \\
$a=1, b=0$ & $\frac{1}{2}-\epsilon$ & $\frac{1}{2}-\epsilon$ \\
$a=0, b=1$ & $\frac{1}{2}-\epsilon$ & $\frac{1}{2}-\epsilon$ \\
$a=1, b=1$ & $\epsilon$ & $\epsilon$ \\
\hline \hline
\end{tabular}


Comparing either of the two columns of the tables (both columns are equal, since they are independent of $\chi$ ), it is clear that they are more distinguishable that either of the two columns of the initial tables. Indeed, using Eqs. (C2), we compute the left-hand side of (18):

$$
\begin{aligned}
S_{\mathrm{b}}\left(\boldsymbol{P}_{\mathrm{f}} \| \boldsymbol{P}_{\mathrm{f}}^{\prime}\right) & =\max _{\chi} S\left(\boldsymbol{P}_{\mathrm{f}}(\cdot, \cdot \mid \chi) \| \boldsymbol{P}_{\mathrm{f}}^{\prime}(\cdot, \cdot \mid \chi)\right) \\
& =S\left(\boldsymbol{P}_{\mathrm{f}}(\cdot, \cdot \mid 0) \| \boldsymbol{P}_{\mathrm{f}}^{\prime}(\cdot, \cdot \mid 0)\right) \\
& =\sum_{a, b} P_{\mathrm{f}}(a, b \mid 0) \log \left(\frac{P_{\mathrm{f}}(a, b \mid 0)}{P_{\mathrm{f}}^{\prime}(a, b \mid 0)}\right) \\
& =\sum_{a, b} P_{0}(a, b \mid b) \log \left(\frac{P_{0}(a, b \mid b)}{P_{0}^{\prime}(a, b \mid b)}\right) \\
& =2\left(\frac{1}{2}-2 \epsilon\right) \log \left(\frac{\frac{1}{2}-\epsilon}{\epsilon}\right) \\
& =2 S_{\mathrm{b}}\left(\boldsymbol{P}_{0} \| \boldsymbol{P}_{0}^{\prime}\right),
\end{aligned}
$$

with $S_{\mathrm{b}}\left(\boldsymbol{P}_{0} \| \boldsymbol{P}_{0}^{\prime}\right)$ given by Eq. (C1). As we see, the RE between the final behaviors doubles the RE between the initial ones.

As a final remark, we note that similar examples can be found for behaviors with larger alphabets. Interestingly, there, WPICC wiring s can increase the behavior RE unboundedly. This can be seen with the following example, which is a generalization of the one above. Consider now an alphabet with $r_{A}=2, r_{B}=s_{A}=k$, and $s_{B}=1$, where $k$ is an arbitrary even number. We defin e $\boldsymbol{P}_{0}$ and $\boldsymbol{P}_{0}^{\prime}$ as

$$
\begin{gathered}
P_{0}(a, b \mid x)= \begin{cases}1 / k-\epsilon & \text { if } a+[b]_{2}=0, \\
\epsilon & \text { if } a+[b]_{2}=1,\end{cases} \\
P_{0}^{\prime}(a, b \mid x)= \begin{cases}P(a, b \mid x) & \text { if } b \neq x, \\
P^{\prime}(a \oplus 1, b \mid x) & \text { if } b=x,\end{cases}
\end{gathered}
$$

where $\epsilon \leqslant \frac{1}{2 k}$. One can easily verify that these behaviors are no signalling a nd well normalized. An analogous calculation to the one of $(\mathrm{C} 1)$ yields

$$
S_{b}\left(\boldsymbol{P}_{0} \| \boldsymbol{P}_{0}^{\prime}\right)=\left(\frac{1}{k}-2 \epsilon\right) \log \left(\frac{\frac{1}{k}-\epsilon}{\epsilon}\right) .
$$

We apply now the same wiring as before, yielding equations (C2) in this case for values of $a, b$ and $\chi \in[k]$. This yields the final behaviors

$$
\begin{aligned}
& P_{f}(a, b \mid \chi)= \begin{cases}1 / k-\epsilon & \text { if } a+[b]_{2}=0, \\
\epsilon & \text { if } a+[b]_{2}=1,\end{cases} \\
& P_{f}^{\prime}(a, b \mid \chi)= \begin{cases}\epsilon & \text { if } a+[b]_{2}=0, \\
1 / k-\epsilon & \text { if } a+[b]_{2}=1 .\end{cases}
\end{aligned}
$$

The final behavior RE is given by

$$
S_{b}\left(\boldsymbol{P}_{f} \| \boldsymbol{P}_{f}^{\prime}\right)=k\left(\frac{1}{k}-2 \epsilon\right) \log \left(\frac{\frac{1}{k}-\epsilon}{\epsilon}\right)=k S_{b}\left(\boldsymbol{P}_{0} \| \boldsymbol{P}_{0}^{\prime}\right) .
$$

Taking for instance $\epsilon=\frac{1}{4 k}$ we find that $S_{b}\left(\boldsymbol{P}_{0} \| \boldsymbol{P}_{0}^{\prime}\right) \rightarrow 0$ as $k$ increases (which means that for large values of $k$ they become indistinguishable), while $S_{b}\left(\boldsymbol{P}_{f} \| \boldsymbol{P}_{f}^{\prime}\right)$ remains constant as $k$ increases.

\section{APPENDIX D: PROOF OF THEOREM 8}

Let us first see that $S_{\mathrm{nl}}$ is a LOSR monotone. This follows from Theorem 2, which implies that $S_{\mathrm{b}}$ is contractive under LOSR wirings, together with Definition 5 and property (6). By virtue of Lemma 6, this implies that $S_{\mathrm{nl}}$ is also a WPICC monotone. Next, let us show that $S_{\mathrm{nl}}$ is convex, which follows from the convexity of $S_{\mathrm{b}}$.

Proof. (Proof of convexity of $S_{\mathrm{nl}}$ ). Let $\overline{\boldsymbol{P}}^{*}=\overline{\boldsymbol{P}}^{*}(\mu)$ be such that $S_{\mathrm{nl}}\left(\mu \boldsymbol{P}+(1-\mu) \boldsymbol{P}^{\prime}\right)=S_{\mathrm{b}}\left(\mu \boldsymbol{P}+(1-\mu) \boldsymbol{P}^{\prime} \| \overline{\boldsymbol{P}}^{*}\right)$, i.e., the optimal local behavior minimizing Eq. (21). Equivalently, let $\boldsymbol{P}^{*}$ and $\boldsymbol{P}^{* *}$ be the optimal local behavior minimizing $S_{\mathrm{nl}}(\boldsymbol{P})$ and $S_{\mathrm{nl}}\left(\boldsymbol{P}^{\prime}\right)$, respectively. Then

$$
\begin{aligned}
S_{\mathrm{nl}} & \left(\mu \boldsymbol{P}+(1-\mu) \boldsymbol{P}^{\prime}\right) \\
: & =S_{\mathrm{b}}\left(\mu \boldsymbol{P}+(1-\mu) \boldsymbol{P}^{\prime} \| \overline{\boldsymbol{P}}^{*}\right) \\
\leqslant & S_{\mathrm{b}}\left(\mu \boldsymbol{P}+(1-\mu) \boldsymbol{P}^{\prime} \| \mu \boldsymbol{P}^{*}+(1-\mu) \boldsymbol{P}^{\prime *}\right) \\
\leqslant & \max _{x, y \in[s]}\left[\mu S\left(\boldsymbol{P}(\cdot, \cdot \mid x, y) \| \boldsymbol{P}^{*}(\cdot, \cdot \mid x, y)\right)\right. \\
& \left.+(1-\mu) S\left(\boldsymbol{P}^{\prime}(\cdot, \cdot \mid x, y) \| \boldsymbol{P}^{\prime *}(\cdot, \cdot \mid x, y)\right)\right] \\
\leqslant & \mu S_{\mathrm{b}}\left(\boldsymbol{P} \| \boldsymbol{P}^{*}\right)+(1-\mu) S_{\mathrm{b}}\left(\boldsymbol{P}^{\prime} \| \boldsymbol{P}^{\prime *}\right) \\
= & \mu S_{\mathrm{nl}}(\boldsymbol{P})+(1-\mu) S_{\mathrm{nl}}\left(\boldsymbol{P}^{\prime}\right),
\end{aligned}
$$

where (D1) follows from Eq. (16) and joint convexity of $S$ and (D2) from Eq. (16) and from maximizing the values of $x$ and $y$ for each term independently.

\section{APPENDIX E: PROOF THAT $S_{\mathrm{nl}}=S_{\mathrm{c}}$}

Note that, since the simplex of generic bipartite probability distributions $\boldsymbol{D}$ is a convex set, von Neumman minimax theorem implies that the order of the maximization and the minimization in Eq. (23c) is irrelevant [24]. That is, for any $\boldsymbol{P}_{\mathrm{NL}} \in \mathrm{NL}$,

$$
\begin{aligned}
S_{\mathrm{c}}\left(\boldsymbol{P}_{\mathrm{NL}}\right): & =\max _{\boldsymbol{D}} \min _{\boldsymbol{P}_{\mathrm{L} \in \mathrm{L}}} S\left(\boldsymbol{P}_{\mathrm{NL}} \cdot \boldsymbol{D} \| \boldsymbol{P}_{\mathrm{L}} \cdot \boldsymbol{D}\right) \\
& =\min _{\boldsymbol{P}_{\mathrm{L}} \in \mathrm{L}} \max _{\boldsymbol{D}} S\left(\boldsymbol{P}_{\mathrm{NL}} \cdot \boldsymbol{D} \| \boldsymbol{P}_{\mathrm{L}} \cdot \boldsymbol{D}\right) \\
& =\min _{\boldsymbol{P}_{\mathrm{L} \in \mathrm{L}}} S_{\mathrm{b}}\left(\boldsymbol{P}_{\mathrm{NL}} \| \boldsymbol{P}_{\mathrm{L}}\right),
\end{aligned}
$$

where Eq. (16) has been used in the last equality. As evident from Eq. (21), the last term is precisely $S_{\mathrm{nl}}\left(\boldsymbol{P}_{\mathrm{NL}}\right)$.

\section{APPENDIX F: PROOF OF THEOREM 9}

The proof is by construction. That is, we find a concrete behavior $\boldsymbol{P}_{0} \in \mathrm{Q}$ and a concrete wiring $\mathcal{W}_{\text {LOSR }} \in$ LOSR, such that $S_{\mathrm{u}}\left(\boldsymbol{P}_{\mathrm{f}}\right)>S_{\mathrm{u}}\left(\boldsymbol{P}_{0}\right)$, where $\boldsymbol{P}_{\mathrm{f}}=\mathcal{W}_{\text {LosR }}\left(\boldsymbol{P}_{0}\right)$.

To this end, let us first consider the Bell scenario where $s=s_{f}=4$ and $r=r_{f}=2$, i.e., four inputs and two outputs for both initial and final behaviors. There, we take $\boldsymbol{P}_{0}$ as equal to the so-called Tsirelson box, for input values $x, y \in\{0,1\}$, and to the white-noise uniform distribution, for input values $x, y \in\{2,3\}$. That is, the components of $\boldsymbol{P}_{0}$ are 


\begin{tabular}{lccc}
\hline \hline$P_{0}(a, b \mid x, y)$ & $x \times y=0$ & $x \times y=1$ & $x \vee y>1$ \\
\hline$a=0, b=0$ & $\frac{p}{2}$ & $\frac{1-p}{2}$ & $\frac{1}{4}$ \\
$a=1, b=0$ & $\frac{1-p}{2}$ & $\frac{p}{2}$ & $\frac{1}{4}$ \\
$a=0, b=1$ & $\frac{1-p}{2}$ & $\frac{p}{2}$ & $\frac{1}{4}$ \\
$a=1, b=1$ & $\frac{p}{2}$ & $\frac{1-p}{2}$ & $\frac{1}{4}$ \\
\hline
\end{tabular}

where $p=\frac{1}{2}+\frac{1}{2 \sqrt{2}}$. A possible physical realization of this behavior is to have Alice and Bob perform adequate quantum measurements on a maximally entangled two-qubit state for two inputs and simply output a random bit for the other two inputs.

Then, as our exemplary wiring, we consider $\mathcal{W}_{\text {LOSR }}$ given by Eq. (7) with the input box chosen as

$$
I^{(\mathrm{L})}(x, y \mid \chi, \psi)= \begin{cases}\delta(x, \chi) \delta(y, \psi) & : \text { if } \chi \times \psi \leqslant 1, \\ \delta\left(x,|\chi|_{2}\right) \delta\left(y,|\psi|_{2}\right) & : \text { if } \chi \times \psi>1,\end{cases}
$$

where $\delta$ stands for the Kronecker $\delta$ and ||$_{2}$ for modulo 2 , and the output box chosen as $O^{(\mathrm{L})}(\alpha, \beta \mid a, b, x, y, \chi, \psi)=$ $\delta(\alpha, a) \delta(\beta, b)$ for all $x, y, \chi$, and $\psi$. Applying this map to $\boldsymbol{P}_{0}$ gives the final behavior $\boldsymbol{P}_{\mathrm{f}}$ of elements

\begin{tabular}{lcc}
\hline \hline$P_{f}(\alpha, \beta \mid \chi, \psi)$ & $\chi, \psi \in \mathcal{S}_{1}$ & $\chi, \psi \in \mathcal{S}_{2}$ \\
\hline$\alpha=0, \beta=0$ & $\frac{p}{2}$ & $\frac{1-p}{2}$ \\
$\alpha=1, \beta=0$ & $\frac{1-p}{2}$ & $\frac{p}{2}$ \\
$\alpha=0, \beta=1$ & $\frac{1-p}{2}$ & $\frac{p}{2}$ \\
$\alpha=1, \beta=1$ & $\frac{p}{2}$ & $\frac{1-p}{2}$ \\
\hline \hline
\end{tabular}

where the setting sets $\mathcal{S}_{1}:=\{(0,0),(0,1),(0,2),(0,3)$, $(1,0),(1,2),(2,0),(2,1),(2,2),(2,3),(3,0),(3,2)\}$ and $\mathcal{S}_{2}:=$ $\{(1,1),(1,3),(3,1),(3,3)\}$ have been introduced. Note that

$$
\boldsymbol{P}_{\mathrm{f}}(\cdot, \cdot \mid \chi, \psi)=\boldsymbol{P}_{0}(\cdot, \cdot \mid \chi, \psi) \forall \chi, \psi \text { such that } \chi \times \psi \leqslant 1 \text {. }
$$

Next, we find a lower bound to $S_{\mathrm{u}}\left(\boldsymbol{P}_{\mathrm{f}}\right)$ that will be seen to be greater than $S_{\mathrm{u}}\left(\boldsymbol{P}_{0}\right)$ below. To this end, we note, using Eq. (23a), that

$$
\begin{aligned}
& S_{\mathrm{u}}\left(\boldsymbol{P}_{\mathrm{f}}\right) \\
& \quad=\min _{\boldsymbol{P}_{\mathrm{L}} \in \mathrm{L}} \frac{1}{16} \sum_{\chi, \psi} S\left(\boldsymbol{P}_{\mathrm{f}}(\cdot, \cdot \mid \chi, \psi) \| \boldsymbol{P}_{\mathrm{L}}(\cdot, \cdot \mid \chi, \psi)\right) \\
& \quad=\frac{1}{16} \min _{\boldsymbol{P}_{\mathrm{L} \in \mathrm{L}}} \sum_{i=1}^{4} \sum_{(\chi, \psi) \in \mathcal{K}_{i}} S\left(\boldsymbol{P}_{\mathrm{f}}(\cdot, \cdot \mid \chi, \psi) \| \boldsymbol{P}_{\mathrm{L}}(\cdot, \cdot \mid \chi, \psi)\right) \\
& \geqslant \frac{1}{16} \sum_{i=1}^{4} \min _{\boldsymbol{P}_{\mathrm{L}}^{(i)} \in \mathrm{L}} \sum_{(\chi, \psi) \in \mathcal{K}_{i}} S\left(\boldsymbol{P}_{\mathrm{f}}(\cdot, \cdot \mid \chi, \psi) \| \boldsymbol{P}_{\mathrm{L}}^{(i)}(\cdot, \cdot \mid \chi, \psi)\right),
\end{aligned}
$$

where $\mathcal{K}_{1}:=\{(0,0),(0,1),(1,0),(\mathbf{1}, \mathbf{1})\}, \quad \mathcal{K}_{2}:=\{(0,2),(0,3)$, $(1,2),(\mathbf{1}, \mathbf{3})\}, \quad \mathcal{K}_{3}:=\{(2,0),(2,1),(3,0),(\mathbf{3}, \mathbf{1})\}, \quad$ and $\quad \mathcal{K}_{4}:=$ $\{(2,2),(2,3),(3,2),(\mathbf{3}, 3)\}$. The four measurement settings in bold are the ones belonging to the set $\mathcal{S}_{2}$ above. All other settings belong to $\mathcal{S}_{1}$. This, by inspection of the table for
$P_{f}(\alpha, \beta \mid \chi, \psi)$ above, implies that the four terms in (F3) are actually equal. Hence

$$
\begin{aligned}
S_{\mathrm{u}}\left(\boldsymbol{P}_{\mathrm{f}}\right) & \geqslant \frac{1}{4} \min _{\boldsymbol{P}_{\mathrm{L} \in \mathrm{L}}} \sum_{(\chi, \psi) \in \mathcal{K}_{1}} S\left(\boldsymbol{P}_{\mathrm{f}}(\cdot, \cdot \mid \chi, \psi) \| \boldsymbol{P}_{\mathrm{L}}(\cdot, \cdot \mid \chi, \psi)\right) \\
& =\frac{1}{4} \min _{\boldsymbol{P}_{\mathrm{L}} \in \mathrm{L}} \sum_{\chi \times \psi \leqslant 1} S\left(\boldsymbol{P}_{\mathrm{f}}(\cdot, \cdot \mid \chi, \psi) \| \boldsymbol{P}_{\mathrm{L}}(\cdot, \cdot \mid \chi, \psi)\right) .
\end{aligned}
$$

Finally, we find an upper bound to $S_{\mathrm{u}}\left(\boldsymbol{P}_{0}\right)$ that is smaller than the right-hand side of Eq. (F4). For this, it is convenient to introduce a behavior $\tilde{\boldsymbol{P}} \in \mathrm{L}$, in the restricted $r=2=s$ scenario, such that

$$
\begin{aligned}
& \sum_{x \times y \leqslant 1} S\left(\boldsymbol{P}_{0}(\cdot, \cdot \mid x, y) \| \tilde{\boldsymbol{P}}(\cdot, \cdot \mid x, y)\right) \\
& =\min _{\boldsymbol{P}_{\mathrm{L}} \in \mathrm{L}} \sum_{x \times y \leqslant 1} S\left(\boldsymbol{P}_{0}(\cdot, \cdot \mid x, y) \| \boldsymbol{P}_{\mathrm{L}}(\cdot, \cdot \mid x, y)\right) .
\end{aligned}
$$

That is, $\tilde{\boldsymbol{P}} \in \mathrm{L}$ minimizes the (uniform input) $\mathrm{RE}$ with respect to $\boldsymbol{P}_{0}$ for the restricted subset of inputs $(x, y) \in \mathcal{K}_{1}$. With this, we construct an educated guess $\boldsymbol{P}^{*} \in \mathrm{L}$ of the actual optimal local behavior for $\boldsymbol{P}_{0}$ for generic inputs, i.e., the one attaining the minimization that defines $S_{\mathrm{u}}\left(\boldsymbol{P}_{0}\right)$. We define $\boldsymbol{P}^{*}$ so as to coincide with $\tilde{\boldsymbol{P}}$ over the restricted input set $\mathcal{K}_{1}$ and with the white-noise uniform distribution over the other inputs. That is,

$$
P^{*}(a, b \mid x, y):= \begin{cases}\tilde{P}(a, b \mid x, y) & : \text { if } x \times y \leqslant 1, \\ 1 / 4 & : \text { if } x \times y>1,\end{cases}
$$

for all $a, b \in[2]$. Then, it holds that

$$
\begin{aligned}
S_{\mathrm{u}}\left(\boldsymbol{P}_{0}\right):= & \frac{1}{16} \min _{\boldsymbol{P}_{\mathrm{L}} \mathrm{L}} \sum_{x, y} S\left(\boldsymbol{P}_{0}(\cdot, \cdot \mid x, y) \| \boldsymbol{P}_{\mathrm{L}}(\cdot, \cdot \mid x, y)\right) \\
\leqslant & \frac{1}{16} \sum_{x, y} S\left(\boldsymbol{P}_{0}(\cdot, \cdot \mid x, y) \| \boldsymbol{P}^{*}(\cdot, \cdot \mid x, y)\right) \\
= & \frac{1}{16}\left[\sum_{x \times y \leqslant 1} S\left(\boldsymbol{P}_{0}(\cdot, \cdot \mid x, y) \| \boldsymbol{P}^{*}(\cdot, \cdot \mid x, y)\right)\right. \\
& \left.+\sum_{x \times y>1} S\left(\boldsymbol{P}_{0}(\cdot, \cdot \mid x, y) \| \boldsymbol{P}^{*}(\cdot, \cdot \mid x, y)\right)\right] \\
= & \frac{1}{16} \sum_{x \times y \leqslant 1} S\left(\boldsymbol{P}_{0}(\cdot, \cdot \mid x, y) \| \boldsymbol{P}^{*}(\cdot, \cdot \mid x, y)\right) \\
= & \frac{1}{16} \sum_{x \times y \leqslant 1} S\left(\boldsymbol{P}_{0}(\cdot, \cdot \mid x, y) \| \tilde{\boldsymbol{P}}(\cdot, \cdot \mid x, y)\right) \\
= & \frac{1}{16} \min _{\boldsymbol{P}_{\mathrm{L}} \in \mathrm{L}} \sum_{x \times y \leqslant 1} S\left(\boldsymbol{P}_{0}(\cdot, \cdot \mid x, y) \| \boldsymbol{P}_{\mathrm{L}}(\cdot, \cdot \mid x, y)\right),
\end{aligned}
$$

where equality (F7) follows from the fact that $\boldsymbol{P}_{0}$ and $\boldsymbol{P}^{*}$ coincide when $x \times y>1$ (they are both equal to the flat distribution), Eq. (F8) from the fact that, by construction of $\boldsymbol{P}^{*}, \boldsymbol{P}^{*}$ and $\tilde{\boldsymbol{P}}$ coincide over the restricted input set $\mathcal{K}_{1}$, and equality (F9) from the definition of $\tilde{\boldsymbol{P}}$ in Eq. (F5). 
The last step is simply to note, using Eqs. (F2), (F9), and (F4), that

$$
S_{\mathrm{u}}\left(\boldsymbol{P}_{\mathrm{f}}\right) \geqslant 4 S_{\mathrm{u}}\left(\boldsymbol{P}_{0}\right)>S_{\mathrm{u}}\left(\boldsymbol{P}_{0}\right)
$$

which shows the theorem's claim.

\section{APPENDIX G: PROOF OF THEOREM 10}

Here, we show that $S_{\text {uc }}$, as defined in Eq. (23c), is a Bell nonlocality monotone. To this end, we first show that it is a LOSR monotone and then that it is convex. By virtue of Theorem 6 and Definition 7, these two facts prove the theorem.

To show monotonicity, it is convenient to introduce a subclass of LOSR wirings called local wirings, denoted as $\mathrm{LW} \subset \mathrm{LOSR}$. It is composed of all wirings $\mathcal{W}_{\mathrm{LW}}$ explicitly parametrized by $\boldsymbol{P}_{\mathrm{f}}=\mathcal{W}_{\mathrm{LW}}\left(\boldsymbol{P}_{0}\right)$, with

$$
\begin{aligned}
P_{\mathrm{f}}(\alpha, \beta \mid \chi, \psi):= & \sum_{a, b, x, y} O^{(\mathrm{LW})}(\alpha, \beta \mid a, b, x, y, \chi, \psi) \\
& \times P_{0}(a, b \mid x, y) I^{(\mathrm{LW})}(x, y \mid \chi, \psi),
\end{aligned}
$$

where $\boldsymbol{I}^{(\mathrm{LW})}:=\boldsymbol{I}^{(A)} \cdot \boldsymbol{I}^{(B)}$ and $\boldsymbol{O}^{(\mathrm{LW})}:=\boldsymbol{O}^{(A)} \cdot \boldsymbol{O}^{(B)}$ are uncorrelated local boxes composed of independent local behaviors for Alice and Bob:

$$
\begin{aligned}
\boldsymbol{I}^{(A)} & :=\left\{I^{(A)}(x \mid \chi)\right\}_{x \in[s], \chi \in\left[s_{f}\right]}, \\
\boldsymbol{O}^{(A)} & :=\left\{O^{(A)}(\alpha \mid a, x, \chi)\right\}_{\alpha \in\left[r_{f}\right], a \in[r], x \in[s], \chi \in\left[s_{f}\right]}, \\
\boldsymbol{I}^{(B)} & :=\left\{I^{(B)}(y \mid \psi)\right\}_{y \in[s], \psi \in\left[s_{f}\right]}, \\
\boldsymbol{O}^{(B)} & :=\left\{O^{(B)}(\beta \mid b, \psi, y)\right\}_{\beta \in\left[r_{f}\right], b \in[r], y \in[s], \psi \in\left[S_{f}\right] .}
\end{aligned}
$$

Then, the following fact is true.

Lemma 11 (LW monotonicity of $S_{\mathrm{uc}}$ ). Let $\boldsymbol{P} \in \mathrm{NS}$ be any no-signaling behavior. Then

$$
S_{\mathrm{uc}}\left(\mathcal{W}_{\mathrm{LW}}(\boldsymbol{P})\right) \leqslant S_{\mathrm{uc}}(\boldsymbol{P})
$$

for all $\mathcal{W}_{\mathrm{LW}} \in \mathrm{LW}$

The proof of this lemma is completely analogous to the one of Theorem 2.

In addition, using Eqs. (4) and (7), one immediately proves the following fact.

Lemma 12. Every $\mathcal{W}_{\mathrm{LOSR}} \in \mathrm{LOSR}$ can be decomposed as

$$
\mathcal{W}_{\mathrm{LOSR}}=\sum_{\lambda} p(\lambda) \mathcal{W}_{\mathrm{LW}}^{(\lambda)}
$$

where $\mathcal{W}_{\mathrm{LW}}^{(\lambda)} \in \mathrm{LW}$ for all $\lambda$.

Note that, if $S_{\mathrm{uc}}$, Lemmas 11 and 12 together imply LOSR monotonicity of $S_{\mathrm{uc}}$. So, the only missing ingredient is to show convexity of $S_{\mathrm{uc}}$, which we do next.

Lemma 13 (Convexity of $S_{\mathrm{uc}}$ ). The statistical strength $S_{\mathrm{uc}}$ is convex. That is,

$$
S_{\mathrm{uc}}\left(\mu \boldsymbol{P}+(1-\mu) \boldsymbol{P}^{\prime}\right) \leqslant \mu S_{\mathrm{uc}}(\boldsymbol{P})+(1-\mu) S_{\mathrm{uc}}\left(\boldsymbol{P}^{\prime}\right),
$$

for all $0 \leqslant \mu \leqslant 1$ and all $\boldsymbol{P}, \boldsymbol{P}^{\prime} \in \mathrm{NS}$.

The proof of this lemma is totally analogous to th e proof of convexity in Appendix D.
[1] J. S. Bell, On the Einsten Podolsky Rosen paradox, Physics 1, 195 (1964).

[2] N. Brunner, D. Cavalcanti, S. Pironio, V. Scarani, and S. Wehner, Bell nonlocality, Rev. Mod. Phys. 86, 419 (2014).

[3] J. Barrett, L. Hardy, and A. Kent, No Signaling and Quantum Key Distribution, Phys. Rev. Lett. 95, 010503 (2005).

[4] A. Acín, N. Gisin, and L. Masanes, From Bell's Theorem to Secure Quantum Key Distribution, Phys. Rev. Lett. 97, 120405 (2006).

[5] A. Acín, N. Brunner, N. Gisin, S. Massar, S. Pironio, and V. Scarani, Device-Independent Security of Quantum Cryptography Against Collective Attacks, Phys. Rev. Lett. 98, 230501 (2007).

[6] R. Colbeck, Ph.D. dissertation, Univ. Cambridge, 2007, arXiv:0911.3814.

[7] S. Pironio et al., Random numbers certified by Bell's theorem, Nature (London) 464, 1021 (2010).

[8] R. Colbeck and R. Renner, Free randomness can be amplified, Nat. Phys. 8, 450 (2012).

[9] R. Gallego, L. Masanes, G. de la Torre, C. Dhara, L. Aolita, and A. Acín, Full randomness from arbitrarily deterministic events, Nat. Commun. 4, 2654 (2013).

[10] H. Buhrman, R. Cleve, S. Massar, and R. de Wolf, Non-locality and communication complexity, Rev. Mod. Phys. 82, 665 (2010).
[11] J. Barrett, N. Linden, S. Massar, S. Pironio, S. Popescu, and D. Roberts, Nonlocal correlations as an information-theoretic resource, Phys. Rev. A 71, 022101 (2005).

[12] J. Allcock, N. Brunner, N. Linden, S. Popescu, P. Skrzypczyk, and T. Vertesi, Closed sets of non-local correlations, Phys. Rev. A 80, 062107 (2009).

[13] B. Lang, T. Vertesi, and M. Navascués Closed sets of correlations: answers from the zoo, J. Phys. A 47, 424029 (2014).

[14] S. Beigi and A. Gohari, A monotone measure for non-local correlations, IEEE Trans. Inf. Theory 61, 5185 (2015).

[15] R. Gallego, L. E. Würflinger, A. Acín, and M. Navascués, An Operational Framework for Nonlocality, Phys. Rev. Lett. 109, 070401 (2012).

[16] P. Joshi, A. Grudka, K. Horodecki, M. Horodecki, P. Horodecki, and R. Horodecki, No-broadcasting of non-signaling boxes via operations which transform local boxes into local ones, QIC 13, 567 (2013).

[17] J. I. de Vicente, On nonlocality as a resource theory and nonlocality measures, J. Phys. A: Math. Thm. 47, 424017 (2014).

[18] K. Horodecki, A. Grudka, P. Joshi, W. Kłobus, and J. Łodyga, Axiomatic approach to contextuality and nonlocality, Phys. Rev. A 92, 032104 (2015).

[19] R. Horodecki, P. Horodecki, M. Horodecki and K. Horodecki, Quantum entanglement, Rev. Mod. Phys. 81, 865 (2009). 
[20] R. Gallego and L. Aolita, Resource Theory of Steering, Phys. Rev. X 5, 041008 (2015).

[21] F. G. S. L. Brandão, M. Horodecki, J. Oppenheim, J. M. Renes, and R. W. Spekkens, Resource Theory of Quantum States Out of Thermal Equilibrium, Phys. Rev. Lett. 111, 250404 (2013).

[22] A. Streltsov, G. Adesso, and M. B. Plenio, Quantum Coherence as a Resource, arXiv:1609.02439.

[23] F. G. S. L. Brandão and G. Gour, Reversible Framework for Quantum Resource Theories, Phys. Rev. Lett. 115, 070503 (2015).

[24] W. van Dam, P. Grunwald, and R. Gill, The statistical strength of nonlocality proofs, IEEE Trans. Inf. Theory 51, 2812 (2005).

[25] A. J. Short, S. Popescu, and N. Gisin, Entanglement swapping for generalized nonlocal correlations, Phys. Rev. A 73, 012101 (2006).

[26] J. Barrett, Information processing in generalized probabilistic theories, Phys. Rev. A 75, 032304 (2007).

[27] We note that, in Ref. [17], the terminology wirings and priorto-input classical communication is used for the class that we call LOSR. The ambiguity originates at the fact that, until now, it was unclear if there could be any difference between both classes of wirings.
[28] S. Kullback and R. A. Leibler, On information and sufficiency, Ann. Math. Stat. 22, 79 (1951).

[29] T. Cover and J. Thomas, Elements of Information Theory (John Wiley \& Sons, Hoboken, NJ, 2006).

[30] H. Chernoff, A measure of asymptotic efficiency for tests of a hypothesis based on the sum of observations, Ann. Math. Stat. 23, 493 (1952).

[31] R. E. Blahut, Hypothesis testing and information theory, IEEE Trans. Inf. Theory 20, 405 (1974).

[32] K. M. R. Audenaert, M. Mosonyi, and F. Verstraete, Quantum state discrimination bounds for finite sample size, J. Math. Phys. 53, 122205 (2012).

[33] C. W. Helström, Quantum Detection and Estimation Theory (Academic Press, New York, 1976).

[34] A. S. Holevo, On asymptotically optimal hypothesis testing in quantum statistics, Theory Probab. Appl. 23, 411 (1978).

[35] V. Vedral and M. B. Plenio, Entanglement measures and purification procedures, Phys. Rev. A 57, 1619 (1998); M. B. Plenio and S. Virmani, An introduction to entanglement measures, Quantum Inf. Comput. 7, 1 (2007).

[36] T. Fritz, Beyond Bell's theorem: correlation scenarios, New J. Phys. 14, 103001 (2012).

[37] D. Bacon and B. F. Toner, Bell Inequalities with Auxiliary Communication, Phys. Rev. Lett. 90, 157904 (2003). 